\title{
Hilbert modular foliations on the projective plane
}

\author{
L. G. Mendes and J. V. Pereira*
}

\begin{abstract}
We describe explicitly holomorphic singular foliations on the projective plane corresponding to natural foliations of Hilbert modular surfaces associated to the field $\mathbb{Q}(\sqrt{5})$. These are concrete models for a very special class of foliations in the recent birational classification of foliations on projective surfaces.
\end{abstract}

Mathematics Subject Classification (2000). Primary 37F75; Secondary 14G35.

Keywords. Holomorphic foliation, Kodaira dimension, Hilbert modular surfaces.

\section{Contents}

1 Introduction and statement of results . . . . . . . . . . . . . . . 244

2 Preliminaries . . . . . . . . . . . . . . . . . . . . . 249

2.1 Hilbert modular surfaces and foliations . . . . . . . . . . . . . . 249

2.2 Birational geometry of Hilbert modular foliations . . . . . . . . . 251

3 Projective models for modular foliations of $Y(5,(2)) \ldots \ldots . . .256$

$3.1 Y(5,(2))$ as Klein's icosahedral surface. . . . . . . . . . . . 256

3.2 Numerical data of the foliations . . . . . . . . . . . . . . . . . 259

3.3 Determining the vector fields . . . . . . . . . . . . . . . 261

3.4 A model where the involution is an automorphism of the plane. . . . 265

4 Projective models for modular foliations of $Y(5) \ldots \ldots \ldots$. . . . . . . 268

4.1 The quotient of $\mathbb{P}^{2}$ by the icosahedral group . . . . . . . . . . . 268

4.2 Numerical data and determination of vector fields . . . . . . . . . 271

5 Projective models for modular foliations of $Y(5,(\sqrt{5})) \ldots \ldots 273$

$5.1 Y(5, \sqrt{5})$ as a double covering of the plane . . . . . . . 273

$5.2 Y(5, \sqrt{5})$ as a modification of $\mathbb{P}^{2}$ and the minimal involution . . . 275

5.3 Numerical data of the foliations . . . . . . . . . . . . . . . 276

A Factorization of the Cremonian involution . . . . . . . . . . 278

B Reduction of singularities of the plane models of $Y(5) \ldots \ldots$. . . . . 282

${ }^{*}$ During the preparation of this paper the authors received financial support respectively from CNPq and Profix-CNPq. 
B.1 Resolution of $\mathscr{H}_{2} \ldots \ldots \ldots \ldots \ldots \ldots \ldots$

B.2 Resolution of $\mathscr{H}_{3} \ldots \ldots \ldots \ldots$. . . . . . . . . . . . 287

\section{Introduction and statement of results}

Our aim is to give concrete models in the projective plane for the holomorphic singular foliations which are the natural foliations of Hilbert modular surfaces.

Such foliations are called Hilbert modular foliations and have a distinguished role in the birational classification of foliations on projective surfaces (cf. [12], [4], [13]). Recently M. Brunella ([5]) and M. McQuillan ([12]) completed the birational classification of holomorphic singular foliations showing that rational fibrations and modular foliations are the unique foliations with negative foliated Kodaira dimension. Besides the role in the birational classification, Hilbert modular foliations have some remarkable dynamical properties and also a distinguished place in the theory of transversally projective foliations.

We recall the definitions. Let $N$ be a square free positive integer, $K$ the totally real quadratic field $\mathbb{Q}(\sqrt{N})$ and $\mathcal{O}_{K}$ the ring of integers of $K$. The two distinct embeddings of $K$ into $\mathbb{R}$ induce an embedding of $\operatorname{PSL}(2, K)$ into $\operatorname{PSL}(2, \mathbb{R}) \times \operatorname{PSL}(2, \mathbb{R})$. If $I \subset \mathcal{O}_{K}$ is a maximal ideal then $\Gamma_{I}$ will be the lattice defined by the following exact sequence:

$$
0 \rightarrow \Gamma_{I} \rightarrow \operatorname{PSL}\left(2, \mathcal{O}_{K}\right) \rightarrow \operatorname{PSL}\left(2, \mathcal{O}_{K} / I\right) \rightarrow 0 .
$$

Denote by $\mathbb{H}^{2}:=\mathbb{H} \times \mathbb{H}$ the product of Poincaré upper planes. Then the Hilbert modular surface $Y(N, I)$ is defined as the minimal desingularization of the compactification of $\mathbb{H}^{2} / \Gamma_{I}$. When the quotient is made by the full Hilbert modular group $\operatorname{PSL}\left(2, \mathcal{O}_{K}\right)$ the associated surface is the Hilbert modular surface $Y(N)$. The Hilbert modular foliations are defined as the singular foliations which are the extensions of the images of the horizontal and vertical fibrations under the quotient defining the Hilbert modular surfaces.

In order to motivate the study of this class of foliations, we list in Theorem 1 some properties of modular foliations. By a reduced foliation we mean a foliation whose singularities are reduced in Seidenberg's sense, see [4]. For the concepts of transversally affine and transversally projective foliations we refer to [16], [17] and references therein.

Theorem 1. Let $\mathscr{H}$ be a reduced modular foliation on the projective surface $S$ and $Z$ the reduced divisor whose support are the invariant algebraic curves of $\mathcal{H}$. Then $\mathscr{H}$ has the following properties.

a. Quasi-minimality: The algebraic invariant curves are rational curves and every non-algebraic leaf is dense. 
b. Hyperbolicity: Except for a finite set, every leaf is hyperbolic and simply-connected, i.e., biholomorphic to the Poincare disc.

c. Uniformity: $\mathcal{H}$ is transversally projective outside $Z$ and there exists a neighborhood $U$ of $Z$ such that $\mathscr{H}_{\mid U \backslash Z}$ is transversally affine.

d. Sporadicity: If $L$ is the class of the cotangent line bundle $T_{\mathscr{H}}^{*}$ in $\operatorname{Pic}(S)$, then $\mathscr{H}$ is the unique holomorphic singular foliation $S$ whose class for the cotangent bundle is $L \in \operatorname{Pic}(S)$.

In the realm of modular surfaces some particular cases are rational surfaces, i.e., birational to $\mathbb{P}^{2}$. We will focus on the following three rational Hilbert modular surfaces $Y(5), Y(5,(2))$ and $Y(5,(\sqrt{5}))$.

We first obtain plane models for the Hilbert modular foliations of $Y(5,(2))$. In the statement below by a dicritical point we mean a singularity of foliation having infinitely many local analytic separatrices and radial points are dicritical singularities locally given by $\omega=x d y-y d x+$ h.o.t. $=0$ :

Theorem 2. The Hilbert modular foliations of $Y(5,(2))$ can be obtained from foliations $\mathscr{H}_{5}$ and $\mathscr{H}_{9}$ of the projective plane by means of one blow up at each of sixteen distinct points $p_{1}, \ldots, p_{16}$ in the plane, with the following properties:

i. $\mathscr{H}_{5}$ and a $\mathscr{H}_{9}$ have degrees 5 and 9 , respectively.

ii. $\mathscr{H}_{5}$ has 16 radial points at $p_{1}, \ldots, p_{16}$ and 15 linearizable saddle points; the quotient of eigenvalues of the saddles is $\frac{-3+\sqrt{5}}{2}$.

iii. $\mathscr{H}_{5}$ and $\mathscr{H}_{9}$ have the same invariant algebraic curves, a configuration of 15 straight lines for which $p_{1} \ldots, p_{10}$ are triple points, $p_{11}, \ldots, p_{16}$ are 5-ple points and the 15 saddle-points of $\mathcal{H}_{5}$ or $\mathscr{H}_{9}$ are nodes. Moreover, the analytical type of the singularities of both foliations on the singular points is the same, except for 6 points $p_{11}, \ldots, p_{16}$ which for $\mathscr{H}_{9}$ are analytically equivalent to dicritical points with algebraic multiplicity 3 and Milnor number 11.

iv. $\mathscr{H}_{5}$ and $\mathscr{H}_{9}$ are invariant under the irreducible action of $A_{5}$, the icosahedral group, on the projective plane.

v. There exists an involutive Cremonian transformation of degree 5 which transforms $\mathscr{H}_{5}$ into $\mathscr{H}_{9}$.

Moreover, there exist affine coordinates on the plane, for which one of the invariant lines is the line at infinity, such that $\mathscr{H}_{5}$ is given by the vector field

$$
\begin{aligned}
& x^{\prime}=\left(x^{2}-1\right)\left(x^{2}-(\sqrt{5}-2)^{2}\right)(x+\sqrt{5} y) \\
& y^{\prime}=\left(y^{2}-1\right)\left(y^{2}-(\sqrt{5}-2)^{2}\right)(y+\sqrt{5} x),
\end{aligned}
$$


while $\mathscr{H}_{9}$ is given by

$$
\begin{aligned}
x^{\prime}= & \left(x^{2}-1\right)\left(x^{2}-(\sqrt{5}-2)^{2}\right)\left[(-40+18 \sqrt{5}) y+(-10 \sqrt{5}+20) y^{3}\right. \\
& +4 \sqrt{5} y^{5}+(8 \sqrt{5}-18) x+(-30+12 \sqrt{5}) x y^{2}+20 x y^{4} \\
& \left.+(4-2 \sqrt{5}) x^{2} y+6 \sqrt{5} x^{2} y^{3}+(10-4 \sqrt{5}) x^{3}+2 x^{3} y^{2}\right] \\
y^{\prime}= & \left(y^{2}-1\right)\left(y^{2}-(\sqrt{5}-2)^{2}\right)\left[(-40+18 \sqrt{5}) x+(-10 \sqrt{5}+20) x^{3}\right. \\
& +4 \sqrt{5} x^{5}+(8 \sqrt{5}-18) y+(-30+12 \sqrt{5}) x^{2} y+20 x^{4} y \\
& \left.+(4-2 \sqrt{5}) x y^{2}+6 \sqrt{5} x^{3} y^{2}+(10-4 \sqrt{5}) y^{3}+2 x^{2} y^{3}\right] .
\end{aligned}
$$

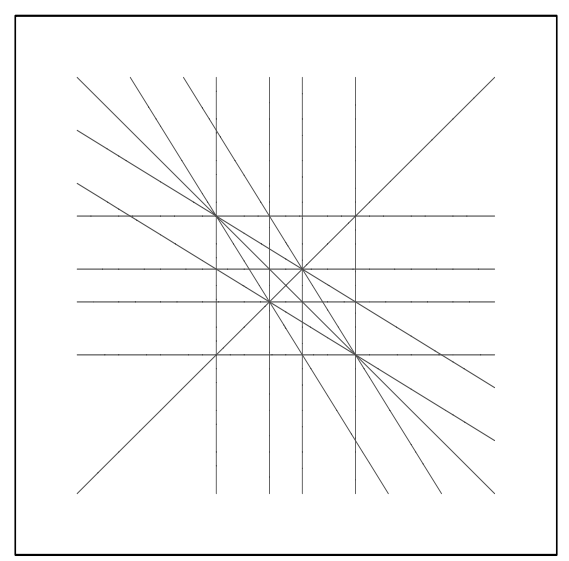

Figure 1. Real picture of the arrangement of lines invariant by $\mathscr{H}_{5}$ and $\mathscr{H}_{9}$. The line at infinity is also invariant.

Remarks on Theorem 2. In Figure 1 there are four directions determined by parallel lines, which correspond to 4 dicritical singularities of $\mathscr{H}_{5}$ and $\mathscr{H}_{9}$ at infinity. Also we remark that the 15 invariant lines determine a simplicial decomposition of $\mathbb{P}_{\mathbb{R}}^{2}$ such that each triangle has one saddle and two dicritical singularities as vertices. Note that both $\mathscr{H}_{5}$ and $\mathcal{H}_{9}$ are induced by real equations and therefore they induce (singular) foliations of $\mathbb{P}_{\mathbb{R}}^{2}$. The qualitative behavior of both of these foliations on the cells of the simplicial decomposition of $\mathbb{P}_{\mathbb{R}}^{2}$ is topologically conjugated to the one presented in Figure 2. It has to be noted that the foliation $\mathscr{H}_{5}$ appeared in [1] as an example of a degree 5 foliation of $\mathbb{R}^{2}$ with the maximum number of invariant lines.

The degree five Cremonian involution sending $\mathscr{H}_{5}$ to $\mathscr{H}_{9}$ in Theorem 2 becomes, after blowing-up the 6 points $p_{11}, \ldots, p_{16}$, an automorphism of a rational surface corresponding to the natural involution of $Y(5,(2))$.

Searching for models in the plane where the involution of $Y(5,(2))$ corresponds to minimal automorphism as defined in [3], we obtain other models in the plane: 


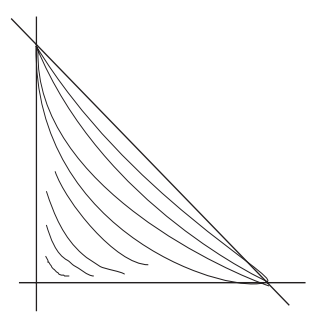

Figure 2. The qualitative behavior of $\mathscr{H}_{5}$ and $\mathscr{H}_{9}$ on each cell.

Theorem 3. The pair of Hilbert modular foliations of $Y(5,(2))$ is birationally equivalent to a pair of foliations $\mathcal{F}, g$ of the projective plane, where both $\mathcal{F}$ and $g$ have degree 7. There exists an involutive automorphism $\phi$ of $\mathbb{P}^{2}$ such that $\phi^{*}(\mathcal{F})=g$ and $\phi^{*}(\mathcal{g})=\mathcal{F}$. Moreover, the algebraic invariant curves are 7 straight lines and 4 conics.

We remark that by the same methods used in the proof of Theorem 3 we can produce another model for the modular foliations of $Y(5,(2))$ in the plane for which both have degree 10 .

Our next result is a detailed description of the Hilbert modular foliations of $Y(5)$, which is regarded as the desingularized quotient $\mathbb{P}^{2} / A_{5}$ (cf. [11]). In the statement below by a minimal reduction of singularities of a foliation we mean a sequence of blow ups in which blow ups at reduced singularities or at regular points of foliations are not allowed.

Theorem 4. The modular foliations of $Y(5)$ are obtained by means of the minimal reduction of singularities of the foliations $\mathscr{H}_{2}$ and $\mathscr{H}_{3}$ of the projective plane with the following properties.

i. The degrees of $\mathscr{H}_{2}$ and $\mathscr{H}_{3}$ are 2 and 3 , respectively, and there are affine coordinates $(x, y)$ where they are induced respectively by

$$
\begin{aligned}
& x^{\prime}=y+32 x-36 x^{2} \\
& y^{\prime}=80 y-60 x y-80 x^{2}
\end{aligned}
$$

and

$$
\begin{aligned}
x^{\prime} & =4 y-3 x y-4 x^{2} \\
y^{\prime} & =-5 y^{2}+80 x y-240 x^{3} .
\end{aligned}
$$

ii. The tangency set of $\mathscr{H}_{2}$ and $\mathscr{H}_{3}$ is an invariant curve composed by the line at infinity and the rational quintic given in the coordinates $(x, y)$ by

$$
Q:-1728 x^{5}+720 x^{3} y-80 x y^{2}+64\left(5 x^{2}-y\right)^{2}+y^{3}=0 .
$$


iii. The non-reduced singularities of $\mathscr{H}_{2}$ and $\mathscr{H}_{3}$ are at the cuspidal points of $Q$, namely: $(0,0),\left(\frac{32}{27}, \frac{1024}{81}\right)$ and the point at infinity $(0,0)=(w, s)=\left(\frac{x}{y}, \frac{1}{y}\right)$, which are dicritical singularities for both foliations.

iv. The sequence of blow ups $\sigma: Y(5) \rightarrow \mathbb{P}^{2}$ producing the reduction of singularities offoliations is composed by eleven blow ups: four blow ups at $(0,0)$ and infinitely near points; four at the point at infinity $(0,0)=(w, s)$ and infinitely near points; three at $\left(\frac{32}{27}, \frac{1024}{81}\right)$ and infinitely near points.

v. The singularities of both foliations at $(1,4)=(x, y)$ are reduced saddles with quotient of eigenvalues equal to $\frac{-3+\sqrt{5}}{2}$.

In Figure 3 we represent (qualitatively) the cuspidal quintic curve.

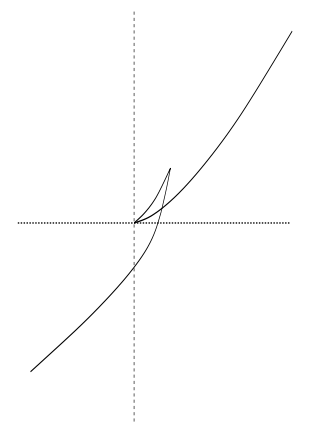

Figure 3. A cuspidal quintic curve and the line at infinity are the tangency set of $\mathscr{H}_{2}$ and $\mathscr{H}_{3}$.

Next, we consider the Hilbert modular foliations on $Y(5,(\sqrt{5}))$. Through a similar analysis to the one made in the proof of Theorem 3 we obtain:

Theorem 5. The pair of Hilbert modular foliations of $Y(5,(\sqrt{5}))$ is birationally equivalent to a pair of foliations $\mathcal{F}, g$ of the projective plane, where both $\mathcal{F}$ and $g$ have degree 9 . There exists an involutive automorphism $\phi$ of $\mathbb{P}^{2}$ such that $\phi^{*}(\mathcal{F})=g$ and $\phi^{*}(\mathcal{G})=\mathcal{F}$. Moreover, the algebraic invariant curves are 5 straight lines and 7 conics.

Again, by the same methods, we can give a second model in the plane for the modular foliations of $Y(5,(\sqrt{5}))$ where the degree is 12 for both modular foliations and the algebraic invariant curves are 1 straight line and 12 conics.

Acknowledgments. The authors thank Karl Otto Stöhr for calling their attention to the work of Hirzebruch on Hilbert modular surfaces. They also thank E. Ghys and the participants of the Seminar of Complex Dynamics at IMPA for the interest and enthusiasm concerning this work. The first author heartly thanks the attention of S. Cantat, D. Cerveau, C. Favre, S. Lamy, J.-M. Lion, L. Meersseman and F. Touzet. 
Remark. After this paper was completed and being submitted, the authors found a paper by R. Kobayashi and I. Naruki, Math. Ann. 279 (1988), 485-500, where they describe the 1 -forms inducing $\mathscr{H}_{5}$ and $\mathscr{H}_{9}$ in another coordinate system. Their interest is in the uniformization theory of surfaces and they do not give a detailed description of the pair of foliations. So we think that our description, by completely different methods, gives new information on the foliations of $Y(5,(2))$. They also describe explicitely the irreducible 2-web produced by the 2-fold ramified covering $p: Y(5,(\sqrt{5})) \rightarrow \mathbb{P}^{2}$, which is complementary to our description of the foliations of $Y(5,(\sqrt{5}))$.

\section{Preliminaries}

2.1. Hilbert modular surfaces and foliations. After having recalled the definition of modular surfaces in the Introduction, let us say a few words about the compactification $\overline{\mathbb{H}^{2} / \Gamma_{I}}$ of $\mathbb{H}^{2} / \Gamma_{I}$. The two embeddings of $K$ into the reals induce an embedding of $\mathbb{P}_{K}^{1}$ into $\mathbb{P}_{\mathbb{R}}^{1} \times \mathbb{P}_{\mathbb{R}}^{1} \subset \mathbb{P}_{\mathbb{C}}^{1} \times \mathbb{P}_{\mathbb{C}}^{1}$. PSL $(2, \mathbb{R})$ acts on $\mathbb{P}_{\mathbb{C}}^{1}$ by fractional linear transformations, thus so does PSL $(2, K)$ on $\mathbb{P}_{K}^{1}$.

The action of the lattice $\Gamma_{I} \subset \operatorname{PSL}(2, K) \subset \operatorname{PSL}(2, \mathbb{R}) \times \operatorname{PSL}(2, \mathbb{R})$ preserves $\mathbb{P}_{K}^{1} \subset \mathbb{P}_{\mathbb{C}}^{1} \times \mathbb{P}_{\mathbb{C}}^{1}$. The orbits of $\mathbb{P}_{K}^{1}$ under the action of $\Gamma_{I}$ are called cusps.

The compactification of $\mathbb{H}^{2} / \Gamma_{I}$ is then obtained by adding the cusps, i.e.,

$$
\overline{\mathbb{H}^{2} / \Gamma_{I}}=\mathbb{H}^{2} / \Gamma_{I} \cup \mathbb{P}_{K}^{1} / \Gamma_{I} .
$$

There exists a bijection between the cusps of $Y(N)$ and the ideal class group of $K$ (see Proposition 1.1 in [7]). In the particular case where the class number of $K$ is one, equivalently $\mathcal{O}_{K}$ is factorial, the cusps of $Y(N, I)$ are parametrized by the finite projective space $\mathbb{P}_{\mathbb{F}}^{1}$, where $\mathbb{F}$ is the finite field $\mathcal{O}_{K} / I$. From (1) we see that the group $\operatorname{PSL}\left(2, \frac{\mathcal{O}_{K}}{I}\right)$ acts on $Y(N, I)$. Of course this action must preserve the cusps and when the cusps of $Y(N, I)$ are parametrized by $\mathbb{P}_{\mathbb{F}}^{1}$, the induced action on $\mathbb{P}_{\mathbb{F}}^{1}$ is the usual action. The modular forms induce a structure of projective, resp. quasi-projective, varieties on $\overline{\mathbb{H}^{2} / \Gamma_{I}}$, resp. $\mathbb{H}^{2} / \Gamma_{I}$. For more details the reader can consult the two first chapters of [7].

As already defined, the Hilbert modular foliations are the singular foliations which are extensions of the images of the horizontal and vertical fibrations under the quotient defining the Hilbert modular surfaces. The algebraic curves introduced in the compactification and desingularization defining the modular surfaces are invariants for both modular foliations.

Along this section we prove Theorem 1, except for the proof of the sporadicity property of modular foliations. This one is postponed to Section 2.2.4, since it depends on notions from the birational classification of foliations which we will recall in 2.2. 
2.1.1. Quasi-minimality. Let $\Gamma \subset \operatorname{PSL}(2, \mathbb{R})^{2}$ be the lattice defining the Hilbert modular surface. If $\mathcal{F}$ is the horizontal foliation of $\mathbb{H} \times \mathbb{H}$ and

$$
\rho: \mathbb{H} \times \mathbb{H} \rightarrow Y \cong \mathbb{H}^{2} / \Gamma
$$

is the quotient map, then the density of leaves of $\rho_{*} \mathcal{F}$ on $Y$ is equivalent to the density of $\pi(\Gamma)$ on $\operatorname{PSL}(2, \mathbb{R})$, where $\pi: \operatorname{PSL}(2, \mathbb{R})^{2} \rightarrow \operatorname{PSL}(2, \mathbb{R})$ is the projection on the second factor.

In the case $\Gamma$ is the image of $\operatorname{PSL}\left(2, \mathcal{O}_{K}\right)$ on $\operatorname{PSL}(2, \mathbb{R})^{2}$ under the pair of embeddings of the totally real quadratic field $K, \pi(\Gamma)$ contains the elements

$$
A_{\mu}=\left[\begin{array}{cc}
1 & \mu \\
0 & 1
\end{array}\right] \text { and } B_{\mu}=\left[\begin{array}{ll}
1 & 0 \\
\mu & 1
\end{array}\right]
$$

for arbitrary $\mu \in \mathcal{O}_{K}$. Let $G$ denote the closure of $\operatorname{PSL}\left(2, \mathcal{O}_{K}\right)$ in $\operatorname{PSL}(2, \mathbb{R})$. Since $\mathcal{O}_{K}$ is dense in $\mathbb{R}$ the Lie algebra of $G$ contains the elements

$$
X=\left[\begin{array}{ll}
0 & 1 \\
0 & 0
\end{array}\right] \text { and } Y=\left[\begin{array}{ll}
0 & 0 \\
1 & 0
\end{array}\right] .
$$

Since $[X, Y]$ is linearly independent of $X$ and $Y$ the Lie algebra of $G$ has dimension 3 , and since $\operatorname{PSL}(2, \mathbb{R})$ is a connected 3-dimensional Lie group, we conclude that $\overline{\operatorname{PSL}\left(2, \mathcal{O}_{K}\right)}=\operatorname{PSL}(2, \mathbb{R})$.

The general case follows from Margulis-Selberg's Theorem, which asserts that any lattice $\Gamma \subset \operatorname{PSL}(2, \mathbb{R})^{2}$ is commensurable with $\Gamma_{K}$ for some totally real quadratic field $K$, i.e., there exists $g \in \operatorname{PSL}(2, \mathbb{R})^{2}$ such that $g \Gamma g^{-1} \cap \Gamma_{K}$ is of finite index in both $\Gamma_{K}$ and $g \Gamma g^{-1}$.

2.1.2. Hyperbolicity. Keeping the notation of the previous section we are going to prove that all the leaves of $\rho_{*} \mathcal{F}$ are hyperbolic and, except for a finite number of exceptions, simply-connected.

The hyperbolicity is obvious since the leaves of $\rho_{*} \mathcal{F}$ are presented as quotient of the upper half-plane $\mathbb{H}$. To conclude that the generic leaf is simply-connected, observe that the non-trivial elements in the fundamental group of a leaf are in correspondence with fixed points of the action of some element of $\pi(\Gamma)$ on $\mathbb{H}$. As $\Gamma$ is discrete we can see that the fixed points are countable, and since to any element of $\pi(\Gamma)$ there correspond at most two fixed points, we may conclude that the generic leave is simply-connected. To conclude observe that every non-simply connected leave must pass through a quotient singularity and the finiteness of the number of non-simply connected leaves follows.

2.1.3. Uniformity. Since the foliation $\rho_{*} \mathcal{F}$ is described as a quotient of $\mathbb{H}^{2}$ by $\Gamma$ we can easily see that every element of the pseudogroup of holonomy of $\rho_{*} \mathcal{F}$ is 
conjugated to a projective transformation, given by the action of $\operatorname{PSL}\left(2, \mathcal{O}_{K}\right)$ on the second factor of $\mathbb{H}^{2}$; thus $\rho_{*} \mathcal{F}$ is transversely projective on the complement of $Z$ in the sense of [16], [17].

In order to understand the local structure of $\rho_{*} \mathcal{F}$ in neighborhood of infinity, i.e. in the neighborhood of the cusps, one has to analyze the structure of the isotropy group of the cusp.

Again, Margulis-Selberg's Theorem allows us to reduce to the case where $\Gamma=$ $\operatorname{PSL}\left(2, \mathcal{O}_{K}\right)$ for some quadratic field $K$. If $\sigma$ is a cusp of $\Gamma$ then the isotropy group of $\sigma$ under the action of $\Gamma$ is conjugated, inside $\Gamma$, to a group of type $G(M, V)$,

$$
G(M, V)=\left\{\left[\begin{array}{cc}
\epsilon & \mu \\
0 & 1
\end{array}\right] \in \operatorname{PSL}(2, K) \mid \epsilon \in V, \mu \in M\right\}=M \rtimes V,
$$

where $M \subset K$ is an additive subgroup of $K$ which has rank 2 as a free abelian group and $V \subset U_{K}^{+}$is a subgroup of the positive units such that for every $\epsilon \in V$ we have $\epsilon M=M$. Therefore the isotropy group of the cusp is an affine group and on neighborhood of infinity of the $Y, \rho_{*} \mathcal{F}$ is transversally affine. Observe also that the orbits are not locally dense.

\subsection{Birational geometry of Hilbert modular foliations}

2.2.1. Kodaira dimension of foliations. In this section we recall the concepts of the birational theory of holomorphic foliations on projective surfaces that we will use along the paper. The references for this section are [12], [4] and [13].

A holomorphic foliation $\mathcal{F}$ on a compact complex surface $S$ is given by an open covering $\left\{U_{i}\right\}$ and holomorphic vector fields $X_{i}$ over each $U_{i}$ such that whenever the intersection of $U_{i}$ and $U_{j}$ is non-empty there exists an invertible holomorphic function $g_{i j}$ satisfying $X_{i}=g_{i j} X_{j}$. The collection $\left\{\left(g_{i j}\right)^{-1}\right\}$ defines a holomorphic line-bundle, called the tangent bundle of $\mathcal{F}$ and denoted $T_{\mathcal{F}}$. The dual of $T_{\mathcal{F}}$ is the cotangent bundle $T_{\mathcal{F}}^{*}$.

Similarly, a holomorphic foliation $\mathcal{F}$ on a compact complex surface $S$ can be given by an open covering $\left\{U_{i}\right\}$ and holomorphic 1-forms $\omega_{i}$ over each $U_{i}$ such that whenever the intersection of $U_{i}$ and $U_{j}$ is non-empty there exists an invertible holomorphic function $h_{i j}$ satisfying $\omega_{i}=h_{i j} \omega_{j}$. The collection $\left\{\left(h_{i j}\right)\right\}$ defines a holomorphic line-bundle, called the normal bundle of $\mathcal{F}$ and denoted $N_{\mathcal{F}}$. The dual of $N_{\mathcal{F}}$ is the conormal bundle $N_{\mathcal{F}}^{*}$.

Along the paper, a foliation means a holomorphic foliation with a finite number of singularities of a smooth projective surface. Observe that there is no loss of generality since every codimension one component of the singular set can be eliminated by factoring out its defining equations from the local vector fields, or 1-forms, inducing the foliations. 
A reduced foliation $\mathcal{F}$ is a foliation such that every singularity $p$ is reduced in Seidenberg's sense, i.e., for every vector field $X$ generating $\mathcal{F}$ and every singular point $p$ of $X$, the eigenvalues of the linear part of $X$ are not both zero and their quotient, when defined, is not a positive rational number. For a reduced foliation $\mathcal{F}$, $T_{\mathcal{F}}^{*}$ is called the foliated canonical bundle and is denoted by $K_{\mathcal{F}}$.

We define the Kodaira dimension $\operatorname{kod}(\mathcal{F})$ of a foliation $\mathcal{F}$ as follows.

Definition 1. If $\mathcal{F}$ is a reduced holomorphic foliation on a projective surface $S$ then

$$
\operatorname{kod}(\mathcal{F}):=\limsup _{n \rightarrow \infty} \frac{\log h^{0}\left(S,\left(K_{\mathcal{F}}\right)^{\otimes n}\right)}{\log n}
$$

When $\mathcal{F}$ is not reduced we $\operatorname{set} \operatorname{kod}(\mathscr{F})$ as $\operatorname{kod}(\overline{\mathcal{F}})$, where $\overline{\mathcal{F}}$ is any reduced foliation birationally equivalent to $\mathcal{F}$

In principle it is necessary to prove that the above definition is well-posed. In fact this is done in [4], [13], [12].

The birational classification of foliations is built on the interplay of two birational invariants of foliations, the above defined foliated Kodaira dimension and the numerical Kodaira dimension. This concept is based on Miyaoka's semipositivity theorem and the Zariski decomposition of pseudo-effective $\mathbb{Z}$-divisors.

Miyaoka's semipositivity theorem ([12], [4]) states that $T_{\mathcal{F}}^{*}$ is a pseudo-effective line bundle (divisor) for any foliation on any projective surface, except for pencils of rational curves (i.e. foliations which after blow ups are rational fibrations). By pseudoeffective we mean a divisor with non-negative intersection with any nef divisor. By nef we mean a divisor whose intersection with any curve is non-negative.

The Zariski decomposition of a pseudo-effective divisor $D$ (or of the associated holomorphic line bundle) is the numerical decomposition of $D$ as $P+N$, where $N$ is a $\mathbb{Q}^{+}$-divisor whose support (possibly empty) is contractible to a normal singularity of surface, $P$ is a nef $\mathbb{Q}$-divisor and $P \cdot N_{i}=0$ for any irreducible component on the support of $N$.

Definition 2. Let $\mathcal{F}$ be a reduced foliation on the complex surface $S$. If $T_{\mathcal{F}}^{*}$ is not pseudo-effective then the numerical Kodaira dimension of $\mathcal{F}$, denoted by $v(\mathcal{F})$, is $-\infty$. Otherwise, if $T_{\mathcal{F}}^{*}=P+N$ is the Zariski's decomposition then we set

$$
v(\mathcal{F})= \begin{cases}0 & \text { when } P \text { is numerically equivalent to zero, } \\ 1 & \text { when } P^{2}=0 \text { but } P \text { is not numerically equivalent to zero, } \\ 2 & \text { when } P^{2}>0 .\end{cases}
$$

When $\mathcal{F}$ is not reduced we set $\nu(\mathcal{F}):=v(\overline{\mathcal{F}})$, where $\overline{\mathcal{F}}$ is any reduced foliation birationally equivalent to $\mathcal{F}$. 
Again, in order to verify the well-posedness of the above definition the reader should consult [4], [12].

A foliation $\mathcal{F}$ of the surface $S$ is relatively minimal if $\mathcal{F}$ is reduced and the contraction of any -1-curve induces a non-reduced foliation on the blow-down of $S$. If $\mathcal{F}$ is a relatively minimal foliation, it is proven in [12] (see also [4]) that the support of the divisor $N$ of the Zariski decomposition of $T_{\mathcal{F}}^{*}$ is composed by chains of $\mathcal{F}$-invariant rational curves of self-intersection lower than -1 . The chain starts with a curve $C^{(1)}$ with just one singularity of the foliation and, if it has more than one component, continues with curves $C^{(k)}$ with 2 singularities. Every singularity in the support of the negative part admits a local holomorphic first integral.

2.2.2. Birational characterization of Hilbert modular foliations. Both notions of Kodaira dimension and numerical dimension can be extended to any line bundle (or divisor) $D$, cf. [15] and there is the general inequality $\operatorname{kod}(D) \leq v(D)$. When $\operatorname{kod}(D)=v(D)$ we usually say that abundance holds for $D$.

The classification result of [12] (see also [5]) asserts that for any foliation $\mathcal{F}$ of projective surface $\operatorname{kod}(\mathcal{F})=v(\mathcal{F})$, except uniquely for the modular foliations, which are birationally characterized by $\operatorname{kod}(\mathcal{F})=-\infty$ and $v(\mathcal{F})=1$. In other terms, $\mathcal{F}$ is a modular foliation if, and only if, abundance does not hold for $K_{\mathcal{F}}$.

We remark that our work gives also concrete examples of (nef) divisor $D$ with $\operatorname{kod}(D)=-\infty$ and $v(D)=1$, for which $D \cdot K_{M}>0$ (a class of examples that, as far we know, does not appear in the literature [15], [2]). The examples consist in taking $D:=K_{\mathcal{H}_{5}}$ or $D:=K_{\mathcal{H}_{9}}$ for the modular foliations of Theorem 2 (details are given in Section 3).

2.2.3. Birational modifications and numerical data. In order to be able to translate the information from the birational characterization of modular foliations into numerical data about singularities and degrees of their projective models, we need to understand the effect of sequences of blowing ups on foliations of the plane.

Definition 3. Let $\mathcal{F}$ be a holomorphic foliation on a surface $S$ and $p \in \operatorname{sing}(\mathcal{F})$. Let $\omega$ be a holomorphic 1-form generating $\mathcal{F}$ on a neighborhood of $p$ and $\pi: \bar{S} \rightarrow S$ the blow-up at $p$.

a. The order of the first non-zero jet of $\omega$ will be denoted by $m(p, \mathcal{F})$. The nonnegative integer $m(p, \mathcal{F})$ is called the algebraic multiplicity of $p$.

b. The vanishing order of $\pi^{*} \omega$ over the exceptional divisor will be denoted by $l(p, \mathcal{F})$.

The above defined indices are related as follows: when the exceptional divisor $E=\pi^{-1}(p)$ is not invariant by $\pi^{*} \mathcal{F}$ then $l(p, \mathcal{F})=m(p, \mathcal{F})+1$; otherwise $l(p, \mathcal{F})=m(p, \mathcal{F})$. More generally when the exceptional divisor $E=\pi^{-1}(p)$ is 
not invariant by the transformed foliation, then

$$
l(p, \mathcal{F})=\operatorname{tang}\left(E, \sigma^{*}(\mathcal{F})\right)+2,
$$

where $\operatorname{tang}\left(E, \pi^{*}(\mathcal{F})\right)$ is the number of tangency points, counted with multiplicities, and when $E=\pi^{-1}(p)$ is $\pi^{*}(\mathcal{F})$-invariant then

$$
l(p, \mathcal{F})=Z(E, \overline{\mathcal{F}})-1,
$$

where $Z(E, \overline{\mathcal{F}})$ denotes the sum of Poincaré-Hopf indices along $E$ of local holomorphic vector fields inducing $\overline{\mathcal{F}}$.

If $\sigma$ is a composition of blow ups $\sigma_{i}$ then cotangent line bundles of the transformed foliation $\overline{\mathcal{F}}$ on $\bar{M}$ and of $\mathcal{F}$ on $M$ are related by

$$
T_{\overline{\mathcal{F}}}^{*}=\sigma^{*}\left(T_{\mathcal{F}}^{*}\right) \otimes \mathcal{O}_{\bar{M}}\left(-\sum_{i}\left(l\left(p_{i}, \mathcal{F}\right)-1\right) \cdot E_{i}\right),
$$

where $\sigma$ denotes the composition of blow ups $\sigma_{i}$ and $E_{i}=\sigma^{-1}\left(p_{i}\right)$. Here we consider the total transforms, i.e., $E_{i}^{2}=-1$ and $E_{i} \cdot E_{j}=0$ if $i \neq j$.

The conormal bundle $N_{\mathcal{F}}^{*}$ of $\mathcal{F}$ on a surface $M$ can be determined by means of the adjunction formula

$$
T_{\mathcal{F}}^{*} \otimes N_{\mathcal{F}}^{*}=K_{M},
$$

where $K_{M}$ is the canonical divisor of $M$. From this relation, the previous isomorphism and the formula

$$
K_{\bar{M}}=\sigma^{*}\left(K_{M}\right) \otimes \mathcal{O}_{\bar{M}}\left(\sum_{i} E_{i}\right),
$$

we obtain for the normal bundle

$$
N_{\overline{\mathcal{F}}}=\sigma^{*}\left(N_{\mathcal{F}}\right) \otimes \mathcal{O}_{\bar{M}}\left(-\sum_{i} l\left(p_{i}, \mathcal{F}\right) \cdot E_{i}\right) .
$$

On $\mathbb{P}^{2}$, the degree $d(\mathcal{F})$ of a foliation $\mathcal{F}$ is defined as the number of tangencies between $\mathcal{F}$ and a generic straight line $L$, counted with multiplicities. There is the following isomorphism:

$$
\left.T_{\mathcal{F}}^{*}=\mathcal{O}_{\mathbb{P}^{2}}(d(\mathcal{F})-1)\right),
$$

and from $K_{\mathbb{P}^{2}}=\mathcal{O}(-3)$ we obtain in the plane $N_{\mathcal{F}}=\mathcal{O}(d(\mathcal{F})+2)$ ).

From the previous remarks and formulae, we can deduce the behavior of the cotangent and normal bundles of foliations of the plane under any finite sequence of blowing ups. Since birational transformations are compositions of blowing ups and blowing downs, it is natural that along this paper an exceptional curve $E=\sigma^{-1}(p)$ arises as strict transform $\bar{C}$ of some rational curve $C \subset \mathbb{P}^{2}$ under blowing ups $\sigma^{\prime}$, i.e. 
$\bar{C}=E$. Also, in some cases the foliation $\sigma^{*}(\mathcal{F})$ will be regarded as the transformed foliation by $\sigma^{\prime}$ of another foliation $g$ in the plane, i.e. $\sigma^{*}(\mathcal{F})=\bar{g}=\left(\sigma^{\prime}\right)^{*}(\mathcal{g})$. In such a situation, for computing

$$
l(p, \mathcal{F})=\operatorname{tang}\left(E, \sigma^{*}(\mathcal{F})\right)+2=\operatorname{tang}(\bar{C}, \bar{g})+2,
$$

it will be necessary to know the following relation:

$$
\operatorname{tang}(\bar{C}, \bar{g})=\operatorname{tang}(C, \mathcal{g})-v_{p}(C) \cdot\left(v_{p}(C)+l(p, \mathcal{g})-1\right),
$$

where $v_{p}(C)$ is the algebraic multiplicity of the curve.

2.2.4. Sporadicity of modular foliations. In our approach, after determining the numerical data of the foliations in the plane (i.e. degrees and multiplicities of singularities) we explicitly determine the polynomial vector fields inducing the modular foliations. What justifies the uniqueness of the foliations submitted to the numerical data is the sporadicity property of modular foliations (cf. [13] and [12]). For the reader's convenience, we sketch below the proof of sporadicity, for further details see [13]. This will complete the proof of Theorem 1.

Let $M$ be a projective surface and denote by $\operatorname{Fol}(M, \mathcal{L})$ the set of foliations of $M$ with foliated canonical bundle isomorphic to $\mathcal{L}$, i.e., $\operatorname{Fol}(M, \mathcal{L})=\mathbb{P} H^{0}\left(M, T_{M} \otimes\right.$ $\mathscr{L})$. We call a foliation $\mathcal{F}$ sporadic if $\operatorname{Fol}(M, \mathscr{L})=\{\mathcal{F}\}$. We assert that if $\mathcal{F}$ is a reduced modular foliation, then $\mathcal{F}$ is sporadic.

In fact, suppose, on the contrary, that $\operatorname{Fol}\left(M, T_{\mathcal{F}}^{*}\right) \neq\{\mathcal{F}\}$ and take $g \neq \mathcal{F}$, $g \in \operatorname{Fol}\left(M, T_{\mathscr{F}}^{*}\right)$. Contractions of local holomorphic vector fields inducing $\mathcal{F}$ and local 1-forms inducing $g$ produce functions vanishing along a tangency curve, which is an algebraic curve (possibly with non-reduced components) denoted by $D_{\mathcal{F} g}$. In equivalent terms we have the isomorphism of line bundles $\mathcal{O}\left(-D_{\mathcal{F} g}\right)=T_{\mathcal{F}} \otimes N_{\mathcal{G}}^{*}$ Thus

$$
T_{\mathcal{F}}^{*} \otimes T_{g}^{*}=\mathcal{O}\left(D_{\mathcal{F} g}\right) \otimes K_{M},
$$

i.e., $T_{\mathcal{F}}^{* \otimes 2}=\mathcal{O}\left(D_{\mathcal{F} g}\right) \otimes K_{M}$. As a consequence, $K_{M}^{\otimes 2}$ is not effective: otherwise $T_{\mathcal{F}}^{* \otimes 4}$ is effective, contradicting $\operatorname{kod}(\mathcal{F})=-\infty$. On the other side, $M$ has no (nontrivial) global holomorphic 1-form (the existence would imply a global section either of $T^{*} \mathcal{F}$ or of the cotangent of the companion modular foliation). Thus we can apply Castelnuovo's criterion of rationality of surfaces, to conclude that $M$ is rational. Now we arrive at a contradiction using the following fact from [15] (a proof is also given in [13]): if $D$ is a pseudo-effective divisor of the rational surface $M$, then $K_{M} \otimes \mathcal{O}(D)$ is pseudo-effective if and only if $\operatorname{kod}\left(K_{M} \otimes \mathcal{O}(D)\right) \geq 0$. This concludes the proof. 


\section{Projective models for modular foliations of $Y(5,(2))$}

In this section we first recall Hirzebruch's description of $Y(5,(2))$ as the blown up $\mathbb{P}^{2}$ at points in $\mathbb{P}_{\mathbb{R}}^{2} \subset \mathbb{P}^{2}$ determined by the vertices and centers of faces of an Icosahedron, cf. [9]. After this, we determine the modular foliations in the plane, using the concepts of Section 2.

Next we express the natural involution of the modular surface as a Cremona transformation changing one foliation into the other. We finish the section proving the existence of other models in the plane where the involution is expressed as an automorphism. In particular, for some plane models the modular foliations can have the same degree.

3.1. $Y(5,(2))$ as Klein's icosahedral surface. Along this section we freely use material from Hirzebruch's paper [9] in order to describe the analytic isomorphism between $Y(5,(2))$ and the blown up projective plane.

Some fundamental facts from [9] are: i) $\mathbb{H}^{2} / \Gamma_{2}$ is a smooth complex open surface; ii) its compactification $\overline{\mathbb{H}^{2} / \Gamma_{2}}$ is a projective singular surface, obtained by adding 5 cusps.

The induced action of $\mathrm{SL}_{2}(\mathcal{O}) / \Gamma_{2}$ on $\overline{\mathbb{H}^{2} / \Gamma_{2}}$ permutes cusps, and for this reason the cusps have the same analytical structure. The minimal resolution of singularities of each cusp introduces a cycle composed by three rational curves of $Y(5,(2))$ with self-intersection number -3 .

The diagonal $\left\{z_{1}=z_{2}\right\}$ of $\mathbb{H}^{2}$ becomes a smooth rational curve $\hat{C}$ of $\overline{\mathbb{H}^{2} / \Gamma_{2}}$, passing through exactly 3 of the 5 cusps (remark that $\hat{C}$ minus three points is hyperbolic). Let us denote by $C$ the strict transform of $\hat{C}$ in $Y(5,(2))$. There are 10 pairwise disjoint curves (including $C$ itself) arising from $C$ by the action induced by $\mathrm{SL}_{2}(\mathcal{O}) / \Gamma_{2}$, which we call for short "diagonals" on $Y(5(2))$. Each of these ten diagonals has self-intersection number -1 in $Y(5,(2))$. If we label the cusps by $c_{i}$, $i=0, \ldots, 4$, then each "diagonal" can be identified as $C_{i j}\left(=C_{j i}\right)$, where $i, j$ refer the pair $c_{i}, c_{j}$ of cusps that do not belong to $C_{i j}$. Denote the pairwise disjoint cycles introduced by elimination of the cusps $c_{i}$ by $A_{i} \cup B_{i} \cup C_{i}$. The intersections of the smooth rational curves $C_{i j}, A_{i}, B_{i}, C_{i}$ are the following:

a. $A_{i} \cdot B_{i}=A_{i} \cdot C_{i}=B_{i} \cdot C_{i}=1$ and $A_{i}^{2}=B_{i}^{2}=C_{i}^{2}=-3$, for $i=0, \ldots, 4$;

b. $C_{i j}$ are pairwise disjoint with $C_{i j}^{2}=-1$;

c. each curve $A_{i}, B_{i}, C_{i}$ intersects 2 curves among the $C_{i j}$ and each $C_{i j}$ passes through 3 components of (three) distinct cycles, as shown in Figure 4.

We also refer to the original paper for the computation of the Euler characteristic, which verifies $e(Y(5,(2))=19$. The main fact is: 
Vol. 80 (2005)

Theorem 6 (Hirzebruch). Up to analytic isomorphism, $Y(5,(2))$ is the unique smooth projective surface with Euler number 19 and having a configuration of 25 rational curves with intersections described in a), b), c) and Figure 4.

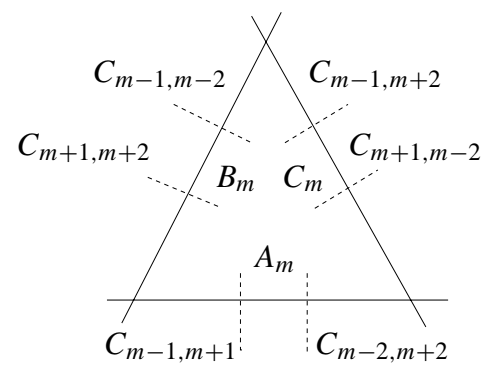

Figure 4. Intersections between diagonals $C_{i j}$ and cycles, with integers taken modulo 5 .

Now we recall Hirzebruch's description of how to obtain such a configuration of rational curves, as in items a), b), c) and Figure 4, by means of 16 blow ups of the projective plane. Consider an icosahedron $I$ in $\mathbb{R}^{3}$ (Figure 5) and denote

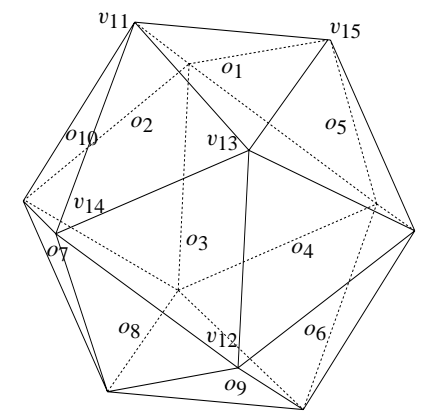

Figure 5. Vertices and centers of faces of an icosahedron.

by $\overline{o_{1}}, \ldots, \overline{o_{20}}$ the points in $\mathbb{R}^{3}$ corresponding to the centers of the 20 faces of $I$. These points can be seen as the vertices of a dual Dodecahedron $D$. Also denote by $\overline{v_{21}}, \ldots, \overline{v_{32}}$ the points in $\mathbb{R}^{3}$ corresponding to the 12 vertices of $I$. Now identify antipodal points among the points $\overline{o_{1}}, \ldots, \overline{v_{32}}$. Denote the 16 points obtained in $\mathbb{P}_{\mathbb{R}}^{2}$ by $o_{1}, \ldots, o_{10}$ and $v_{11}, \ldots, v_{16}$. The 30 edges of $I$ determine 15 straight lines in $\mathbb{P}_{\mathbb{R}}^{2}$, 
denoted $L_{A_{i}}, L_{B_{i}}, L_{C_{i}}$, where $i=0, \ldots, 4$ :

$$
\begin{array}{lll}
L_{A_{0}}:=\overline{v_{12} v_{13}}, & L_{B_{0}}:=\overline{v_{14} v_{16}}, & L_{C_{0}}:=\overline{v_{11} v_{15}}, \\
L_{A_{1}}:=\overline{v_{12} v_{15}}, & L_{B_{1}}:=\overline{v_{11} v_{14}}, & L_{C_{1}}:=\overline{v_{13} v_{16}}, \\
L_{A_{2}}:=\overline{v_{12} v_{16}}, & L_{B_{2}}:=\overline{v_{11} v_{13}}, & L_{C_{2}}:=\overline{v_{14} v_{15}}, \\
L_{A_{3}}:=\overline{v_{12} v_{14}}, & L_{B_{3}}:=\overline{v_{13} v_{15}}, \quad L_{C_{3}}:=\overline{v_{11} v_{16}}, \\
L_{A_{4}}:=\overline{v_{11} v_{12}}, & L_{B_{4}}:=\overline{v_{15} v_{16}}, & L_{C_{4}}:=\overline{v_{13} v_{14}},
\end{array}
$$

Since 5 edges of $I$ intersect at each vertex of $I, 5$ lines of the 15 lines $L_{A_{i}}, L_{B_{i}}$, $L_{C_{i}}$ pass through each point $v_{i}, i=11, \ldots, 16$. Moreover, if 3 mutually orthogonal edges of $I \subset \mathbb{R}^{3}$ are prolonged they intersect at the center of a face of $I$; so we conclude that 3 of the 15 lines pass through each point $o_{i}$. These intersections are given in the tables below (we give the intersections in all details for further use along the paper).

\begin{tabular}{|c|c|}
\hline points & intersection of lines \\
\hline$v_{11}$ & $L_{C_{0}} \cdot L_{B_{1}} \cdot L_{B_{2}} \cdot L_{C_{3}} \cdot L_{A_{4}}$ \\
\hline$v_{12}$ & $L_{A_{0}} \cdot L_{A_{1}} \cdot L_{A_{2}} \cdot L_{A_{3}} \cdot L_{A_{4}}$ \\
\hline$v_{13}$ & $L_{A_{0}} \cdot L_{C_{1}} \cdot L_{B_{2}} \cdot L_{B_{3}} \cdot L_{C_{4}}$ \\
\hline$v_{14}$ & $L_{B_{0}} \cdot L_{B_{1}} \cdot L_{C_{2}} \cdot L_{A_{3}} \cdot L_{C_{4}}$ \\
\hline$v_{15}$ & $L_{C_{0}} \cdot L_{A_{1}} \cdot L_{C_{2}} \cdot L_{B_{3}} \cdot L_{B_{4}}$ \\
\hline$v_{16}$ & $L_{B_{0}} \cdot L_{C_{1}} \cdot L_{A_{2}} \cdot L_{C_{3}} \cdot L_{B_{4}}$ \\
\hline
\end{tabular}

\begin{tabular}{|c|c||c|c|}
\hline points & intersection of lines & points & intersection of lines \\
\hline$o_{1}$ & $L_{A_{0}} \cdot L_{C_{2}} \cdot L_{C_{3}}$ & $o_{2}$ & $L_{C_{1}} \cdot L_{C_{2}} \cdot L_{A_{4}}$ \\
\hline$o_{3}$ & $L_{B_{0}} \cdot L_{B_{3}} \cdot L_{A_{4}}$ & $o_{4}$ & $L_{B_{0}} \cdot L_{A_{1}} \cdot L_{B_{2}}$ \\
\hline$o_{5}$ & $L_{A_{1}} \cdot L_{C_{3}} \cdot L_{C_{4}}$ & $o_{6}$ & $L_{B_{2}} \cdot L_{A_{3}} \cdot L_{B_{4}}$ \\
\hline$o_{7}$ & $L_{C_{0}} \cdot L_{A_{2}} \cdot L_{C_{4}}$ & $o_{8}$ & $L_{B_{1}} \cdot L_{A_{2}} \cdot L_{B_{3}}$ \\
\hline$o_{9}$ & $L_{A_{0}} \cdot L_{B_{1}} \cdot L_{B_{4}}$ & $o_{10}$ & $L_{C_{0}} \cdot L_{C_{1}} \cdot L_{A_{3}}$ \\
\hline
\end{tabular}

We refer to this configuration of fifteen lines $L_{A_{i}}, L_{B_{i}}$ and $L_{C_{i}}$ as the icosahedral configuration of lines, which is represented in Figure 6.

Consider now the inclusion $\mathbb{P}_{\mathbb{R}}^{2} \subset \mathbb{P}^{2}$ and the 15 complex projective lines determined by the icosahedral configuration. Consider the complex surface $M$ obtained by blow up of $\mathbb{P}^{2}$ at the 16 points $o_{1}, \ldots, o_{10}, v_{11}, \ldots, v_{16}$. This complex surface $M$ is the Klein's icosahedral surface. Since one blow up increases the Euler number by one, we have $e(M)=e\left(\mathbb{P}^{2}\right)+16=19$. 


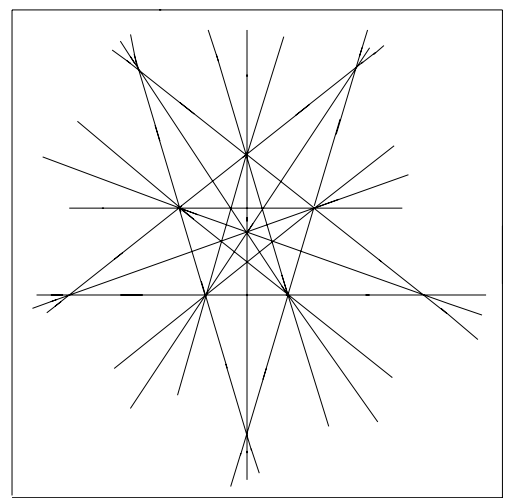

Figure 6. The icosahedral configuration of lines.

Next, consider the strict transforms in $M$ of the complex lines, denoted $\overline{L_{A_{i}}}, \overline{L_{B_{i}}}$, $\overline{L_{C_{i}}}$. Since each complex line received 4 blow ups, we obtain in $M$ :

$$
{\overline{L_{A_{i}}}}^{2}={\overline{L_{B_{i}}}}^{2}={\overline{L_{C}}}^{2}=-3 \text {. }
$$

Denote now by $C_{i j}$ the exceptional lines $E_{k}=\sigma^{-1}\left(o_{k}\right)$, for $k=1, \ldots, 10$, if $o_{k}$ does $n o t$ belong to lines in the icosahedral configuration indexed by $i$ or $j$. The reader can check that the 25 curves $\overline{L_{A_{i}}}, \overline{L_{B_{i}}}, \overline{L_{C_{i}}}, C_{i j}$ have the intersection properties described

in items a), b), c) and Figure 4. Also we can check that $\overline{L_{A_{i}}} \cup \overline{L_{B_{i}}} \cup \overline{L_{C_{i}}}$ are five disjoint cycles in $M$.

\subsection{Numerical data of the foliations}

Proposition 1. Let $\mathcal{F}^{\prime}$ and $g^{\prime}$ be the modular foliations in the plane producing the modular foliations $\mathcal{F}$ and $g$ of $Y(5,(2))$ under the 16 blow ups $\sigma: Y(5,(2)) \rightarrow \mathbb{P}^{2}$ defining $Y(5,(2))$ as Klein's icosahedral surface. Then $\mathcal{F}^{\prime}$ and $g^{\prime}$ have the following properties:

(1) The degree of $\mathcal{F}^{\prime}$ is 5 and the degree of $\mathcal{G}^{\prime}$ is 9.

(2) For every $i \in\{1, \ldots, 10\}$, the algebraic multiplicities are

$$
m\left(o_{i}, \mathcal{F}^{\prime}\right)=m\left(o_{i}, g^{\prime}\right)=1 .
$$

(3) For every $i \in\{11, \ldots, 16\}, m\left(v_{i}, \mathcal{F}^{\prime}\right)=1$ and $m\left(v_{i}, \mathscr{g}^{\prime}\right)=3$.

(4) The $o_{i}$ are radial singularities for $\mathcal{F}^{\prime}$ and $\mathcal{G}^{\prime}$.

(5) The $v_{i}$ are dicritical singularities eliminable by one blow-up for both $\mathcal{F}^{\prime}$ and $\mathscr{g}^{\prime}$; for $\mathcal{F}^{\prime}$ the $v_{i}$ have Milnor number 1, while for $g^{\prime}$ they have Milnor number 11. 
Proof. The singularities of $\mathcal{F}$ and $g$ at the corners of the cycles $A_{i} \cup B_{i} \cup C_{i} \subset$ $Y(5,(2))$ are not affected by the blowing downs producing $\mathbb{P}^{2}$, see Figure 4 . Such singularities at corners are reduced, with Milnor number one. Plugging in $A_{i}^{2}=$ $B_{i}^{2}=C_{i}^{2}=-3$ in Camacho-Sad's formula the quotient of eigenvalues of the singularities of $\mathcal{F}$ and $\mathcal{G}$ is determined and turns out to be equal to $\lambda=\frac{-3 \pm \sqrt{5}}{2}$. These singularities sum 15 reduced singularities, for both $\mathcal{F}^{\prime}$ and $\mathcal{g}^{\prime}$, with the same quotient of eigenvalues.

The 16 blow downs transforming $M=Y(5,(2))$ into $\mathbb{P}^{2}$ include the blowing downs of 10 "diagonals" $C_{i j}$. Since the $C_{i j}$ are completely transverse to both $\mathcal{F}$ and $g$, cf. Figure 4, the transformed foliations $\mathcal{F}^{\prime}$ and $g^{\prime}$ in the plane have radial points at $o_{i}$, with $m\left(o_{i}, \mathcal{F}^{\prime}\right)=m\left(o_{i}, \mathscr{g}^{\prime}\right)=1$ for $i=1, \ldots, 10$.

The points $v_{11}, \ldots, v_{16}$ are also obtained from the blow down of extra $(-1)$ curves which are neither $\mathcal{F}$ - nor $\mathcal{G}$-invariant and that do not pass through the corners of the cycles in $Y(5,(2))$, cf. Figure 4 . Thus $v_{i}(i=11, \ldots, 16)$ are dicritical points for both $\mathcal{F}^{\prime}$ and $g^{\prime}$, with the extra property that the transformed foliations have no singularities along $\sigma_{i}^{-1}\left(v_{i}\right)$.

The automorphism group of $Y(5,(2))$ permutes the cycles resolving the cusps, therefore

$$
m\left(v_{11}, \mathcal{F}^{\prime}\right)=\cdots=m\left(v_{16}, \mathcal{F}^{\prime}\right) \quad \text { and } \quad m\left(v_{11}, \mathscr{g}^{\prime}\right)=\cdots=m\left(v_{16}, g^{\prime}\right) .
$$

Denoting the blow ups producing $Y(5,(2))$ from $\mathbb{P}^{2}$ by $\sigma:=\sigma_{1} \circ \ldots \circ \sigma_{16}$, we have, cf. (2), the isomorphisms

$$
\begin{aligned}
T_{\mathcal{F}}^{*} & =\sigma^{*} \mathcal{O}_{\mathbb{P}^{2}}\left(d\left(\mathcal{F}^{\prime}\right)-1\right) \otimes \mathcal{O}_{M}\left(-\left(\sum_{i=1}^{10} E_{i}-\sum_{i=11}^{16} m\left(v_{i}, \mathcal{F}^{\prime}\right) E_{i}\right),\right. \\
T_{\mathcal{G}}^{*} & =\sigma^{*} \mathcal{O}_{\mathbb{P}^{2}}\left(d\left(\mathcal{G}^{\prime}\right)-1\right) \otimes \mathcal{O}_{M}\left(-\left(\sum_{i=1}^{10} E_{i}-\sum_{i=11}^{16} m\left(v_{i}, \mathcal{G}^{\prime}\right) E_{i}\right),\right.
\end{aligned}
$$

where $E_{i}=\sigma^{-1}\left(o_{i}\right)$ for $i=1, \ldots, 10$ and $E_{i}=\sigma^{-1}\left(v_{i}\right)$ for $i=11, \ldots, 16$. Remark that the 15 reduced singularities are not affected.

The rational invariant curves for $\mathcal{F}$ or $\mathcal{G}$ are the cycles, so the $N$-part for $T_{\mathcal{F}}^{*}$ and $T_{q}^{*}$ is empty, cf. Section 2. Therefore, since the numerical Kodaira dimension is one for modular foliations, we have $T_{\mathcal{F}}^{*} \cdot T_{\mathcal{F}}^{*}=0$ and $T_{\mathrm{g}}^{*} \cdot T_{\mathrm{g}}^{*}=0$, which can be written as

$$
\left(d\left(\mathcal{F}^{\prime}\right)-1\right)^{2}=10+\sum_{i=11}^{16} m\left(v_{i}, \mathcal{F}^{\prime}\right)^{2}=10+6 \cdot m\left(\mathcal{F}^{\prime}\right)^{2}
$$

and

$$
\left(d\left(g^{\prime}\right)-1\right)^{2}=10+\sum_{i=11}^{16} m\left(v_{i}, g^{\prime}\right)^{2}=10+6 \cdot m\left(g^{\prime}\right)^{2}
$$


from which we have $d\left(\mathcal{F}^{\prime}\right) \geq 5$ and $d\left(\mathscr{g}^{\prime}\right) \geq 5$.

The tangency locus of $\mathcal{F}$ and $\mathcal{G}$ on $M=Y(5,(2))$ is the reduced (i.e. free of multiple components) curve given by the union of the five cycles of $M=Y(5,(2))$, cf. [4], [12].

We can write

$$
\mathcal{O}_{M}\left(\sum_{i=0}^{4} A_{i}+B_{i}+C_{i}\right)=\sigma^{*} \mathcal{O}_{\mathbb{P}^{2}}(15) \otimes \mathcal{O}\left(-\sum_{i=1}^{10} 3 E_{i}-\sum_{i=11}^{16} 5 E_{i}\right),
$$

since $o_{i}$ are triple points and $v_{i}$ are 5-uple points in the arrangement of 15 lines. Since the tangency locus is described by a section of $T_{\mathcal{F}}^{*} \otimes N_{\mathrm{g}}$, cf. Section 2,

$$
T_{\mathcal{F}}^{*} \otimes N_{g}=\sigma^{*} \mathcal{O}_{\mathbb{P}^{2}}(15) \otimes \mathcal{O}\left(-\sum_{i=1}^{10} 3 E_{i}-\sum_{i=11}^{16} 5 E_{i}\right) .
$$

Expanding the left hand side of the equation above in terms of the generators $\sigma^{*} \mathcal{O}_{\mathbb{P}^{2}}(1)$, $E_{1}, \ldots, E_{16}$ of $\operatorname{Pic}(M)$ we deduce that $d\left(\mathcal{F}^{\prime}\right)+d\left(\mathcal{g}^{\prime}\right)=14$ and

$$
m\left(v_{i}, \mathcal{F}^{\prime}\right)+m\left(v_{i}, \mathcal{G}^{\prime}\right)=4, \quad \text { for all } i=11, \ldots, 16 .
$$

Since $d\left(\mathcal{F}^{\prime}\right), d\left(\mathscr{g}^{\prime}\right) \geq 5$, the unique possible positive solutions with $d\left(\mathcal{F}^{\prime}\right) \leq d\left(\mathscr{g}^{\prime}\right)$ are

$$
\left(d\left(\mathcal{F}^{\prime}\right), d\left(\mathscr{g}^{\prime}\right)\right) \in\{(5,9),(6,8),(7,7)\} .
$$

The possibilities $\left(d\left(\mathcal{F}^{\prime}\right), d\left(\mathcal{g}^{\prime}\right)\right)=(6,8)$ or $(7,7)$ are excluded by (3) and (4). Therefore $\left(d\left(\mathcal{F}^{\prime}\right), d\left(\mathcal{g}^{\prime}\right)\right)=(5,9)$ and

$$
m\left(v_{11}, \mathcal{F}^{\prime}\right)=\cdots=m\left(v_{16}, \mathcal{F}^{\prime}\right)=1 \quad \text { and } \quad m\left(v_{11}, \mathscr{g}^{\prime}\right)=\cdots=m\left(v_{16}, \mathscr{G}^{\prime}\right)=3 .
$$

To conclude observe that the sum of Milnor numbers for $g^{\prime}$ is $9^{2}+9+1=91$ (Darboux's theorem) and that there are 15 reduced saddles and 10 radial points for $g^{\prime}$, thus $\mu\left(v_{i}, g^{\prime}\right)=11$ for all $i=11, \ldots, 16$.

Remark 1. We will show in Appendix A that there are Cremona maps transforming the pair of modular foliations with degrees 5 and 9 given in this Proposition 1 into pairs of modular foliations with degrees $(6,8)$ and also $(7,7)$. But either the Cremona transformations produce invariant conics or the transformed foliations have singularities no longer eliminable by just one blow up.

3.3. Determining the vector fields. Taking in consideration Proposition 1 , we denote by $\mathscr{H}_{5}$ and $\mathscr{H}_{9}$, respectively, the modular foliations of the plane of degree 5 and of degree 9 . In order to explicitly determine polynomial vector fields inducing the foliations $\mathscr{H}_{5}$ and $\mathscr{H}_{9}$, we will first locate its dicritical singularities in the plane. 
These are the points $o_{1}, \ldots, o_{10}, v_{11}, \ldots, v_{16}$ of Proposition 1 . In order to construct a plane model for this arrangement we follow [6] and take the coordinates in $\mathbb{R}^{3}$ of the 12 vertices of an Icosahedron $I$ (whose edge is 2):

$$
( \pm 1,0, \pm \tau), \quad(0, \pm \tau, \pm 1), \quad( \pm \tau, \pm 1,0),
$$

where $\tau=\frac{1-\sqrt{5}}{2}$ is the golden ratio (recall the basic equation $\tau^{2}=\tau+1$ ). The coordinates of the vertices of the dual Dodecahedron $D$ (with edge $2 \tau^{-1}$ ) are

$$
\left(0, \pm \tau^{-1}, \pm \tau\right), \quad\left( \pm \tau, 0, \pm \tau^{-1}\right), \quad\left( \pm \tau^{-1}, \pm \tau, 0\right), \quad( \pm 1, \pm 1, \pm 1) .
$$

In the projectivization of $\mathbb{R}^{3}$ to $\mathbb{P}_{\mathbb{R}}^{2}$, we determine

$$
\begin{aligned}
& o_{1}=\left(0: \tau^{-1}, \tau\right), \quad o_{2}=(-1: 1: 1), \quad o_{3}=\left(\tau^{-1}:-\tau: 0\right), \\
& o_{4}=\left(\tau^{-1}: \tau: 0\right), \quad o_{5}=(1: 1: 1), \quad o_{6}=(1: 1:-1) \text {, } \\
& o_{7}=\left(\tau: 0: \tau^{-1}\right), \quad o_{8}=(1:-1: 1), \quad o_{9}=\left(0:-\tau^{-1}: \tau\right) \text {, } \\
& o_{10}=\left(-\tau: 0: \tau^{-1}\right) \text {, }
\end{aligned}
$$

and from the vertices of $I$ the following points in $\mathbb{P}_{\mathbb{R}}^{2}$ :

$$
\begin{array}{lll}
v_{11}=(-1: 0: \tau), & v_{12}=(0: \tau:-1), & v_{13}=(0: \tau: 1), \\
v_{14}=(-\tau: 1: 0), & v_{15}=(1: 0: \tau), & v_{16}=(\tau: 1: 0) .
\end{array}
$$

Let us determine convenient affine coordinates for the singularities of $\mathscr{H}_{5}$ and $\mathscr{H}_{9}$. Take affine coordinates $(\bar{x}, \bar{y}):=(X: Y: 1)$, for which the line $L_{B_{0}}:=\overline{v_{14} v_{16}}$ in the icosahedral configuration becomes the line at infinity.

So $o_{3}, o_{4}, v_{14}, v_{16}$ become points at infinity and this produces four parallel directions for some of the affine lines of the icosahedral configuration (Figure 6), which now is represented in Figure 7.

From these affine coordinates $(\bar{x}, \bar{y})$ we obtain after a change of affine coordinates, given by

$$
(x, y):=\left(\begin{array}{cc}
\tau & \tau+1 \\
-\tau & \tau+1
\end{array}\right) \cdot(\bar{x}, \bar{y}),
$$

the affine coordinates desired in $\mathbb{R}^{2}$, for which the lines in the arrangement are those given in Figure 1 in the Introduction: four horizontal lines $y= \pm 1$ and $y= \pm(2 \tau+1)$ and four vertical lines $x= \pm 1$ and $x= \pm(2 \tau+1)$. The coordinates $(x, y)$ for the singular points are shown in Table 1.

The strategy now is to associate to the general polynomial vector field $X=$ $X(x, y)$ of degree 5 a system of linear equations in its coefficients in such a way that the solutions of this linear system correspond to foliations of degree 5 with $o_{1}, \ldots, o_{10}$ and $v_{11}, \ldots, v_{16}$ as radial singularities. 


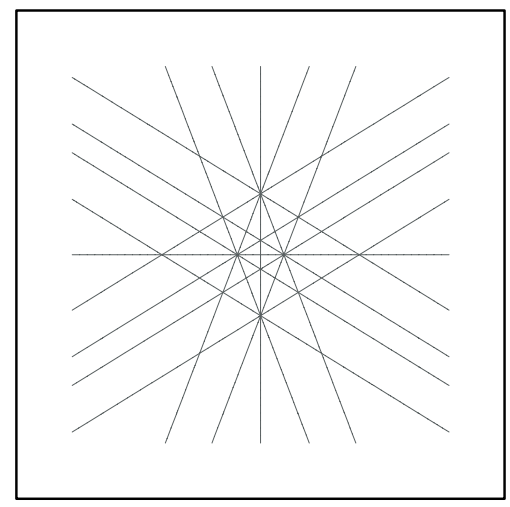

Figure 7. The arrangement of lines induced by the icosahedron, with one line at infinity.

Table 1. Affine coordinates for the singularities, where $\tau=\frac{1-\sqrt{5}}{2}$ is the golden ratio.

\begin{tabular}{|l|c|l|}
\hline$o_{1}=(1,1)$ & $o_{2}=(1,2 \tau+1)$ & $o_{5}=(2 \tau+1,1)$ \\
\hline$o_{6}=(-2 \tau-1,-1)$ & $o_{7}=(-2 \tau-1,2 \tau+1)$ & $o_{8}=(-1,-2 \tau-1)$ \\
\hline$o_{9}=(-1,-1)$ & $o_{10}=(-2 \tau-1,2 \tau+1)$ & $v_{11}=(-1,1)$ \\
\hline$v_{12}=(-2 \tau-1,-2 \tau-1)$ & $v_{13}=(2 \tau+1,2 \tau+1)$ & $v_{15}=(1,-1)$, \\
\hline
\end{tabular}

Let $g$ be a foliation corresponding to a solution of this linear system. Let $L$ be any line of the configuration. Suppose that $L$ is not $g$-invariant. Then, since $g$ has 4 radial singularities on $L$, the order of tangency between $g$ and $L$ is at least 8 . This is in contradiction with the fact that $g$ has degree 5 . Then any line $L$ of the configuration must be invariant by $g$. From this we deduce that $g$ is also the unique solution, since with any other solution the tangency locus of $g$ should have degree 11 and contains the 15 lines of the configuration. Hence to determine $\mathscr{H}_{5}$ we found the unique solution of the linear system mentioned above, obtaining the result stated in Theorem 2 . The computations were carried out with the help of a computer algebra system.

To determine $\mathscr{H}_{9}$ we can repeat the same strategy, i.e., writing down the linear system in the coefficients of the generic foliation of degree 9 whose solutions corresponds to foliations with radial singularities on $o_{1}, \ldots, o_{10}$ and singularities with algebraic multiplicity at least 3 on $v_{11}, \ldots, v_{16}$. In order to reduce the number of indeterminates we use the fact that any solution to our problem must be written in the form

$$
\left(x^{2}-1\right)\left(x^{2}-(2 \tau+1)^{2}\right) P_{5} \frac{\partial}{\partial x}+\left(y^{2}-1\right)\left(y^{2}-(2 \tau+1)^{2}\right) Q_{5} \frac{\partial}{\partial y},
$$

where $P_{5}$ and $Q_{5}$ are generic polynomials of degree 5. This follows from our choice 
of coordinates $(x, y)$ for which the lines $y= \pm 1, y= \pm(2 \tau+1)$ and $x= \pm 1$, $x= \pm(2 \tau+1)$ are invariant by the foliations.

Solving the linear system in the coefficients of $P_{5}$ and $Q_{5}$ leads to the vector field of degree 9 presented in the statement of Theorem 2 .

3.3.1. The involution of $Y(5,(2))$ as a birational transformation of the plane. We will describe the Cremonian transformation $\mathcal{T}$ of the plane to itself transforming $\mathscr{H}_{5}$ into $\mathscr{H}_{9}$, which expresses in the plane the automorphism $I: Y(5,(2)) \rightarrow Y(5,(2))$ sending one modular foliation to the other. More precisely, we will show that, if $\Sigma: N \rightarrow \mathbb{P}^{2}$ denotes the blow up at the six vertices $\left\{v_{11}, \ldots, v_{16}\right\}$, then there exists a Cremonian transformation $\mathcal{T}$ such that $\Sigma \circ I \circ \Sigma^{-1}=\mathcal{T}$, where by abuse of notation $I$ denotes the involution of $N$ sending one modular foliation to the other.

The definition of the involution $\mathcal{T}$ as a birational transformation is the following (cf. [14]). Let $E_{j} \subset N$ be the exceptional lines of the blowing ups of $v_{11}, v_{12}, v_{13}$, $v_{14}, v_{15}, v_{16}$. Consider the 6 conics $C_{p_{1}, \ldots, p_{5}}$ passing through exactly five vertices $p_{i} \in\left\{v_{11}, v_{12}, v_{13}, v_{14}, v_{15}, v_{16}\right\}$. Therefore its strict transforms $\bar{C}_{p_{1}, \ldots, p_{5}} \subset N$ are $(-1)$-curves, because

$$
\bar{C}_{p_{1}, \ldots, p_{5}}^{2}=C_{p_{1}, \ldots, p_{5}}^{2}-5=-1 .
$$

The involution $I: N \rightarrow N$ sends each $\bar{C}_{p_{1}, \ldots, p_{5}}$ to the unique $E_{j}$ which does not intersect it. The six curves $\bar{C}_{p_{1}, \ldots, p_{5}}$ can be blown down to six points in a non-singular surface $N^{\prime}$. Let $\overline{E_{j}} \subset N^{\prime}$ denote the strict transforms of the exceptional lines $E_{j}$ by such blow downs. Since exactly five conics among the $C_{p_{1}, \ldots, p_{5}}$ passes through each vertex $v_{1 j}$, each $E_{j}$ intersects exactly five curves among the $\bar{C}_{p_{1}, \ldots, p_{5}}$, and we conclude that the self-intersections of $\overline{E_{j}}$ in $N^{\prime}$ satisfy

$$
\overline{E_{j}^{2}}=E_{j}^{2}+5=4 \text {. }
$$

Moreover, since $N^{\prime}$ is a rational surface and $e\left(N^{\prime}\right)=e(N)-6=3$, then $N^{\prime}=\mathbb{P}^{2}$ and $\overline{E_{j}}$ is a conic.

It is well known that the surface $N$ obtained from $\mathbb{P}^{2}$ by one blow up at each vertex $\left\{v_{11}, \ldots, v_{16}\right\}$ is embedded in the projective 3 -space as a smooth cubic surface. A smooth cubic surface in 3 -space has exactly 27 straight lines. Among these lines we will find the strict transforms of the 15 lines in the icosahedral arrangement of lines. The remaining 12 lines are given by the set $E_{j}, j=1, \ldots, 6$ and the six curves $\bar{C}_{p_{1}, \ldots, p_{5}}$.

In Appendix A we describe $\mathcal{T}$ as a degree 5 Cremonian transformation $\mathcal{T}=\mathcal{T}_{5}$, which factorizes as a composition of three quadratic birational transformations of the plane:

$$
\mathcal{T}_{5}=Q_{3} \circ Q_{2} \circ Q_{1}
$$


The understanding of this factorization will enable us to give more models of modular foliations, with degrees $6,7,8$, starting from $\mathscr{H}_{5}$.

3.4. A model where the involution is an automorphism of the plane. In the previous section we described the involution $\mathcal{T}$ sending the modular foliation $\mathscr{H}_{5}$ to $\mathcal{H}_{9}$ as a birational transformation. Now we show that we can obtain models in the plane for the Hilbert modular foliations of $Y(5,(2))$ for which the involution is an automorphism of the plane (in particular the pair of foliations has the same degree), that is, we want to prove here Theorem 3 stated in the Introduction.

Let $\Sigma: N \rightarrow \mathbb{P}^{2}$ be the blowing up at the six indetermination points $v_{11}, \ldots, v_{16}$ of $\mathcal{T}$ and consider again the 6 conics $C_{p_{1}, \ldots, p_{5}}$ passing through exactly five points among $v_{11}, \ldots, v_{16}$. Thus $I=\Sigma^{-1} \circ \mathcal{T} \circ \Sigma$ is a non-minimal regular involution in the sense of Bayle-Beauville [3]. This is due to the fact that $\bar{C}_{p_{1}, \ldots, p_{5}}$ is sent to $E_{j}$ and these (-1)-curves are disjoint. Our task now is to obtain from $I: N \rightarrow N$ a minimal involution $\bar{I}$ of $\mathbb{P}_{\mathbb{C}}^{1} \times \mathbb{P}_{\mathbb{C}}^{1}$ and from this an automorphism $L$ of the plane. For this purpose we firstly describe the map $g$ and the birational map $E$ in the next diagram, where $\Gamma$ is the birational transformation stated in Theorem 3:

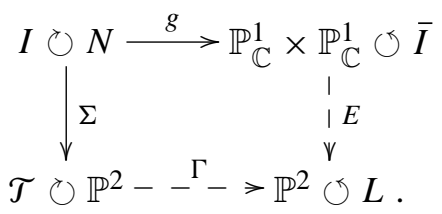

Consider again the lines $L_{A_{i}}, L_{B_{i}}$ and $L_{C_{i}}$ of the icosahedral configuration, and denote now by $\overline{L_{A_{i}}}, \overline{L_{B_{i}}}$ and $\overline{L_{C_{i}}}$ the strict transforms by $\Sigma$. Remark that these are $(-1)$-curves of $N$. Since the involution has order two and there are 5 curves $\overline{L_{A_{i}}}$ in $N$, it can be proved [9] that the effect of the involution is described as

$$
I\left(\overline{L_{A_{1}}}\right)=\overline{L_{A_{1}}}, \quad I\left(\overline{L_{A_{0}}}\right)=\overline{L_{A_{2}}}, \quad I\left(\overline{L_{A_{3}}}\right)=\overline{L_{A_{4}}} .
$$

Consider now the blow down of the 5 curves $\overline{L_{A_{i}}} \subset N$ to points denoted $r_{A_{i}}, i=$ $1, \ldots, 5$ of the resulting smooth surface $W$, denoted $g: N \rightarrow W$. That is, we have chosen to blow down one component of each triangle $\overline{L_{A_{i}}} \cup \overline{L_{B_{i}}} \cup \overline{L_{C_{i}}}$ of $N$. In [9] it is proven that the rational surface $W$, which is minimal (since its Euler number is $e(M)=9-5=4)$, in fact is isomorphic to $\mathbb{P}_{\mathbb{C}}^{1} \times \mathbb{P}_{\mathbb{C}}^{1}$. We can verify that the transformed curves by $g$ of $\overline{L_{B_{i}}}$ and $\overline{L_{C}}$ (we keep the same notation for the curves in $N$ ) have self-intersection equal to 2 in $W=\mathbb{P}_{\mathbb{C}}^{1} \times \mathbb{P}_{\mathbb{C}}^{1}$. For instance, in $N$ we have ${\overline{L_{B}}}^{2}=-1$, but the intersections in $N$ are

$$
\overline{L_{B_{1}}} \cdot \overline{L_{A_{0}}}=\overline{L_{B_{1}}} \cdot \overline{L_{A_{1}}}=\overline{L_{B_{1}}} \cdot \overline{L_{A_{2}}}=1
$$


(as we can check from the tables in Section 3.1), and so by blowing down $\overline{L_{A_{0}}}$, $\overline{L_{A_{1}}}, \overline{L_{A_{2}}}$, the intersection number of the $\overline{L_{B_{1}}}$ in $\mathbb{P}_{\mathbb{C}}^{1} \times \mathbb{P}_{\mathbb{C}}^{1}$ is increased by 3 . So the transformed curves of $\overline{L_{B_{i}}}$ and $\overline{L_{C_{i}}}$ are curves of $\mathbb{P}_{\mathbb{C}}^{1} \times \mathbb{P}_{\mathbb{C}}^{1}$ with self-intersection number 2; so they are curves of bi-degrees $(1,1)$. Besides the point $r_{A_{i}}$ they intersect along an extra point $q$ of $\mathbb{P}_{\mathbb{C}}^{1} \times \mathbb{P}_{\mathbb{C}}^{1}$. The points $r_{A_{i}}$ are dicritical points for the foliations and the extra-point $q$ is a saddle point for the foliations (see Figure 8).

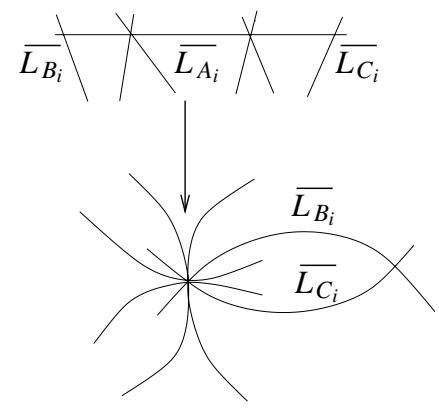

Figure 8. Contraction producing the point $r_{A_{i}}$ of $\mathbb{P}_{\mathbb{C}}^{1} \times \mathbb{P}_{\mathbb{C}}^{1}$.

In coordinates of $\mathbb{P}_{\mathbb{C}}^{1} \times \mathbb{P}_{\mathbb{C}}^{1}$, the involution obtained can be written as

$$
\bar{I}(x, y)=\left(\frac{1}{y}, \frac{1}{x}\right) .
$$

With $\tau=\frac{(1-\sqrt{5})}{2}$, following [9] we put

$$
\begin{gathered}
r_{A_{1}}=(1,1), \quad r_{A_{1}}=(0,0), \quad r_{A_{2}}=(\infty, \infty), \\
r_{A_{3}}=(-\tau, \tau-1), \quad r_{A_{4}}=(-\tau+1, \tau) .
\end{gathered}
$$

The singularities of the pair of foliations $\overline{\mathcal{F}}$ and $\bar{g}$ obtained in $\mathbb{P}_{\mathbb{C}}^{1} \times \mathbb{P}_{\mathbb{C}}^{1}$ are exactly: a) 5 singularities at $r_{A_{i}}$ with Milnor number 9 and algebraic multiplicity $m\left(r_{A_{i}}\right)=3$ (since its blow up produces an invariant exceptional line with two radial points and two reduced singularities) and b) 5 saddle points.

Denoting by $H$ and $V$ horizontal and vertical fibers, we write

$$
\begin{aligned}
T_{\overline{\mathcal{F}}}^{*} & =\mathcal{O}\left(d_{1}(\overline{\mathcal{F}}) H+d_{2}(\overline{\mathcal{F}}) V\right), \\
N_{\overline{\mathcal{F}}} & =\mathcal{O}\left(\left(d_{1}(\overline{\mathcal{F}})+2\right) H+\left(d_{2}(\overline{\mathcal{F}})+2\right) V\right)
\end{aligned}
$$

(analogously for $\bar{g}$ ), and when combined with Darboux's theorem

$$
\sum \mu(\overline{\mathcal{F}}, p)-e\left(\mathbb{P}_{\mathbb{C}}^{1} \times \mathbb{P}_{\mathbb{C}}^{1}\right)=T_{\overline{\mathcal{F}}}^{*} \cdot N_{\overline{\mathcal{F}}}
$$


we obtain

$$
5 \cdot 9+5 \cdot 1-4=2 d_{1}(\overline{\mathcal{F}}) \cdot d_{2}(\overline{\mathcal{F}})+2\left(d_{1}(\overline{\mathcal{F}})+d_{2}(\overline{\mathcal{F}})\right)
$$

hence $d_{1}(\overline{\mathcal{F}}) \cdot d_{2}(\overline{\mathcal{F}})+d_{1}(\overline{\mathcal{F}})+d_{2}(\overline{\mathcal{F}})=23$.

Now we consider the Poincaré-Hopf formula applied to the transformed curve of $\bar{L}_{B_{i}}$ in $\mathbb{P}_{\mathbb{C}}^{1} \times \mathbb{P}_{\mathbb{C}}^{1}$ :

$$
\begin{aligned}
T_{\overline{\mathcal{F}}}^{*} \cdot \bar{L}_{B_{i}} & =T_{\overline{\mathcal{F}}}^{*} \cdot(H+V) \\
& =1+Z\left(r_{L_{A_{i}}}\right)+Z\left(r_{L_{A_{j}}}\right)+Z\left(r_{L_{A_{k}}}\right)-\chi\left(\bar{L}_{B_{i}}\right) \\
& =3 \cdot 3+1-2=8,
\end{aligned}
$$

where $i \neq j \neq k \in\{0,1,2,3,4\}$; so we conclude that $d_{1}(\overline{\mathcal{F}})+d_{2}(\overline{\mathcal{F}})=8$ and

$$
d_{1}(\overline{\mathcal{F}}) \cdot d_{2}(\overline{\mathcal{F}})=23-8=15
$$

from the previous relations. Easily we obtain that the unique solutions for the bidegree $\left(d_{1}(\overline{\mathcal{F}}), d_{2}(\overline{\mathcal{F}})\right)$ are $(3,5)$ and $(5,3)$. If $\mathcal{F}$ has bi-degree $(3,5)$ then the companion modular foliation $\bar{g}$ has bi-degree $(5,3)$, since they are related by the involution of $\bar{I}: \mathbb{P}_{\mathbb{C}}^{1} \times \mathbb{P}_{\mathbb{C}}^{1} \circlearrowleft$.

3.4.1. The birational map $\boldsymbol{E}$. We will consider the birational map $E: \mathbb{P}_{\mathbb{C}}^{1} \times \mathbb{P}_{\mathbb{C}}^{1}$ $-\rightarrow>\mathbb{P}^{2}$, in order to obtain from the foliations with bi-degrees $\left(d_{1}(\overline{\mathcal{F}}), d_{2}(\overline{\mathcal{F}})\right)=$ $(3,5)$ and $\left(d_{1}(\bar{g}), d_{2}(\bar{g})\right)=(5,3)$ a pair of foliations of the plane, both with degree 7 .

Then the so-called elementary transformation $E$ is defined as follows: it is given by blowing up a point $p$ and then contracting the strict transforms of the horizontal and vertical fibers through $p$.

We will compute the degree of foliations in the plane, denoted $\mathcal{F}^{\prime}$ and $\mathcal{g}^{\prime}$, by using the Poincaré-Hopf theorem applied to the straight line $L$ in the plane which is the transform of the exceptional line $E=\sigma^{-1}(p)$. Let us choose $p=r_{A_{1}} \in \mathbb{P}_{\mathbb{C}}^{1} \times \mathbb{P}_{\mathbb{C}}^{1}$, a singularity of both $\overline{\mathcal{F}}$ and $\bar{g}$ (cf. previous section).

If $h=\operatorname{col}(H)$ and $v=\operatorname{col}(V)$ are the dicritical points produced by the blow downs of the transformed curves of the horizontal and vertical fibers, $\bar{H}$ and $\bar{V}$ in Figure 9, then Poincaré-Hopf in the plane yields

$$
d\left(\mathcal{F}^{\prime}\right)-1=\sum_{q \in L} Z\left(q, \mathcal{F}^{\prime}\right)-\chi(L)=4+m\left(h, \mathcal{F}^{\prime}\right)+m\left(v, \mathcal{F}^{\prime}\right)-2,
$$

where 4 is the contribution of two radial points and two reduced points. We can use 


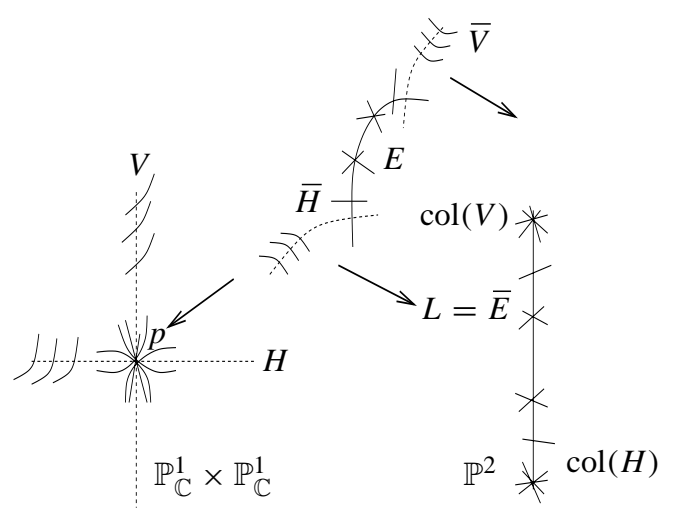

Figure 9. Passing from $\mathbb{P}_{\mathbb{C}}^{1} \times \mathbb{P}_{\mathbb{C}}^{1}$ to $\mathbb{P}^{2}$.

the remarks of Section 2.2.3 for the computation of $m\left(p, \mathcal{F}^{\prime}\right)$, obtaining

$$
\begin{aligned}
d\left(\mathcal{F}^{\prime}\right)-1 & =4+[\operatorname{tang}(\overline{\mathcal{F}}, \bar{H})+1]+[\operatorname{tang}(\overline{\mathcal{F}}, \bar{V})+1]-2 \\
& =4+\left[\left(d_{1}(\mathcal{F})-3\right)+1\right]+\left[\left(d_{2}(\mathcal{F})-3\right)+1\right]-2 \\
& =d_{1}(\mathcal{F})+d_{2}(\mathcal{F})-2,
\end{aligned}
$$

thus $d\left(\mathcal{F}^{\prime}\right)-1=6$ as desired. Since we blow up a point $p$ belonging to 6 of the invariant $(1,1)$ - curves, the pair of modular foliations obtained in the plane has 6 invariant straight lines besides the line $L$ and 4 invariant conics, images of the $(1,1)$-curves not blown up.

Of course, we could have chosen another point $p$ for the elementary transformation. For instance if we choose a point which is regular for both foliations, then the pair of foliations in the plane would have degree 10.

\section{Projective models for modular foliations of $Y(5)$}

4.1. The quotient of $\mathbb{P}^{\mathbf{2}}$ by the icosahedral group. We can regard the quotient in the definition of $Y(5)$ (cf. (1) in the Introduction) as

$$
\mathbb{H}^{2} / \operatorname{PSL}_{2}\left(\mathcal{O}_{K}\right) \cong\left(\mathbb{H}^{2} / \Gamma_{2}\right) / \operatorname{PSL}_{2}\left(\mathcal{O}_{K} /(2)\right),
$$

and it is known that $\operatorname{PSL}_{2}\left(\mathcal{O}_{K} /(2)\right) \cong \operatorname{PSL}_{2}\left(\mathbb{F}_{4}\right) \cong A_{5}$, the icosahedral group. Following the classical approach due to F. Klein, we describe $Y(5)$ as a birational modification of the quotient $S:=\mathbb{P}^{2} / A_{5}$, where $A_{5}$ acts on the plane (which is the blow down of $Y(5,(2)))$. In other words, we consider the modular foliations of $Y(5)$ as the quotients of $\mathscr{H}_{5}$ and $\mathscr{H}_{9}$ of Theorem 2 under their group of symmetries $A_{5}$. 
The facts described in detail in [11] that we use are the following. Let $\pi: \hat{S} \rightarrow S$ be the minimal desingularization of $S=\mathbb{P}^{2} / A_{5}$. Since $A_{5}$ is finite, $\hat{S}$ is birationally equivalent to $\mathbb{P}^{2}$. From Hirzebruch's work we know that the Euler characteristic of $Y(5)$ is 14 and $Y(5)$ can be obtained from the plane $\mathbb{P}^{2}$ by means of 11 blow ups, denoted $f: Y(5) \rightarrow \mathbb{P}^{2}$. Denoting again $\sigma: Y(5,(2)) \rightarrow \mathbb{P}^{2}$ the sixteen blow ups of the Klein icosahedral surface (cf. previous sections), there is the following commutative diagram:

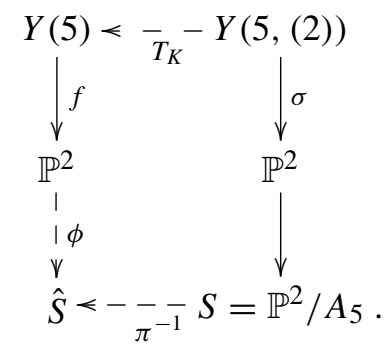

In what follows we exploit the map $f: Y(5) \rightarrow \mathbb{P}^{2}$, but we remark that the explicit coordinates of the rational map $T_{K}$ are known from Klein's work on the ring of invariants for the action of $A_{5}$ on the plane [11], and so the study of $T_{K}$ could be another way to get the modular foliations of $Y(5)$.

The strict transform by the birational transformation $\phi: \mathbb{P}^{2}-->\hat{S}$ of the quotient of the icosahedral arrangement of lines is an irreducible rational quintic curve $Q \subset \mathbb{P}^{2}$. There are affine coordinates $(x, y)$ of $\mathbb{P}^{2}-L_{\infty}$ for which the quintic is $Q:-1728 x^{5}+$ $720 x^{3} y-80 x y^{2}+64\left(5 x^{2}-y\right)^{2}+y^{3}=0$; the line at infinity $L=L_{\infty} \subset \mathbb{P}^{2}$ is the strict transform by $\phi$ of a component of the exceptional divisor introduced by $\pi$.

The eleven points to be blown up by $f: Y(5) \rightarrow \mathbb{P}^{2}$ are the points that must be blown up in order to obtain normal crossing between the strict transform of $Q$ by $f$ and the exceptional divisor of its resolution, see pages 635-636 of [11]. The singularities of the quintic $Q$ are at the points $(0,0),(1,4),\left(\frac{32}{27}, \frac{1024}{81}\right)$ and at infinity $(0,0)=(w, s)=\left(\frac{x}{y}, \frac{1}{y}\right)$. At $(0,0)$ and at infinity the quintic is locally given as $z^{2}-t^{5}=0$, at $\left(\frac{32}{27}, \frac{1024}{81}\right)$ it is locally given as $z^{2}-t^{3}=0$ and at $(1,4) Q$ has a nodal point. The resolution process is done by means of four blow ups at $(0,0)$ (and infinitely near points), four blow ups at infinity $(0,0)=(w, s)$ (and infinitely near points) and three at $\left(\frac{32}{27}, \frac{1024}{81}\right)$ (and infinitely near points), as shown in Figure 10. Some remarks on Figure 10 are useful. We denote by $E_{i}$ the exceptional line of the $i$-th blow up $E_{i}=\sigma_{i}^{-1}\left(p_{i}\right)$, for $i=1, \ldots, 11$. Then we denote by $\overline{E_{i}}$ their strict transforms by subsequent blow ups. For instance, according to Figure 10: $E_{i}^{2}=-1$ for $i \in\{4,8,11\}, \overline{E_{i}^{2}}=-2$, for $i \in\{1,3,5,7,10\}$ and $\overline{L^{2}}=-2, \overline{E_{i}^{2}}=-3$ for $i \in\{2,6,9\}$.

Denoting the sequence of eleven blow ups by $f$, the strict transform of the quintic 


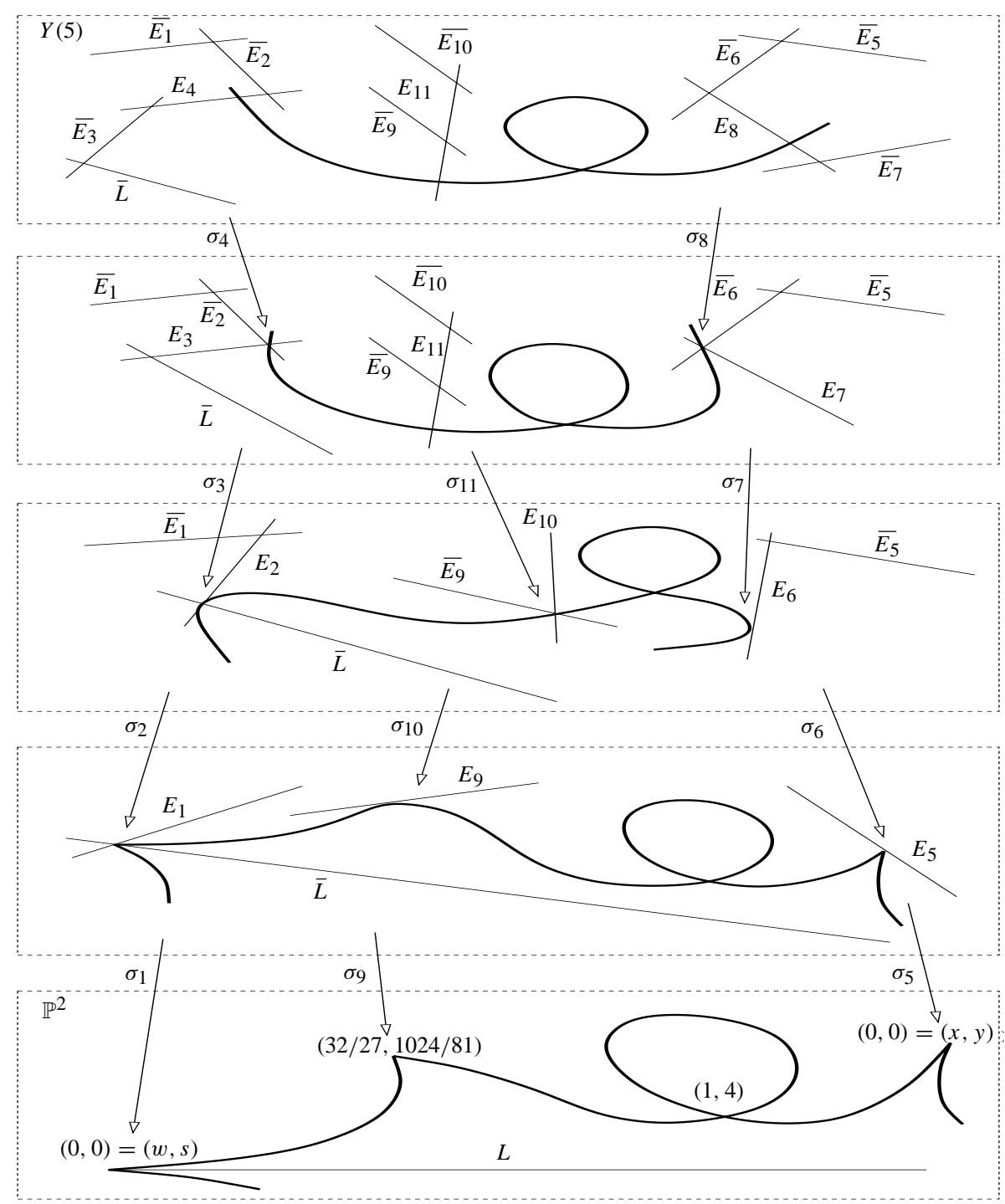

Figure 10. The 11 blow ups composing $f: Y(5) \rightarrow \mathbb{P}^{2}$. 
in $Y(5)$, denoted $\bar{Q}$, can be described $\mathcal{O}(\bar{Q})$ in $\operatorname{Pic}(Y(5)$ as

$f^{*} \mathcal{O}_{\mathbb{P}^{2}}(5) \otimes \mathcal{O}\left(-2 E_{1}-2 E_{2}-E_{3}-E_{4}-2 E_{5}-2 E_{6}-E_{7}-E_{8}-2 E_{9}-E_{10}-E_{11}\right)$.

Hence $\bar{Q}$ is a rational nodal curve with self-intersection $5^{2}-26=-1$, as shown in Figure 11. In $Y(5), \bar{Q}$ corresponds to the desingularization of the unique cusp introduced by compactification of $\mathbb{H}^{2} / \mathrm{PSL}_{2}\left(\mathcal{O}_{K}\right)$.

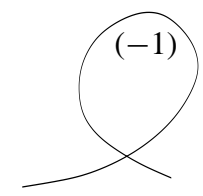

Figure 11. $\bar{Q} \subset Y(5)$ is a cycle introduced in the resolution of the cusp.

4.2. Numerical data and determination of vector fields. Considering $Y(5)$ as the blown up plane by $f$, its pair of modular foliations, denoted $\overline{\mathscr{H}}_{2}$ and $\overline{\mathscr{H}}_{3}$ (the indices 2 and 3 will be justified), are the transformed foliations of foliations in the plane denoted $\mathscr{H}_{2}$ and $\mathscr{H}_{3}$.

The pair $\overline{\mathscr{H}}_{2}$ and $\overline{\mathscr{H}}_{3}$ has as (reduced) tangency curve the following curves: i) the strict transform of the quintic $Q$, denoted $\bar{Q}$, ii) the strict transform of the line at infinity $L$, denoted $\bar{L}$ and iii) the curves $\bar{E}_{i}$, for $i=1,2,3,5,6,7,9,10$.

Remark that $\overline{E_{4}}, \overline{E_{8}}, \overline{E_{11}}$ are not $\mathscr{H}_{2}$ or $\mathscr{H}_{3}$-invariant, since they intersect the cycle $\bar{Q}$ and the unique singularities of these foliations along the cycle are at the node of $\bar{Q}$.

The strict transform of the line at infinity $L$ in $Y(5)$, denoted $\bar{L}$, can be described in $\operatorname{Pic}(Y(5))$ as

$$
\mathcal{O}(\bar{L})=f^{*} \mathcal{O}(1) \otimes \mathcal{O}\left(-E_{1}-E_{2}-E_{3}\right),
$$

and we can easily write $\overline{E_{i}}$ in terms of $E_{i}$ 's, for instance

$$
\overline{E_{1}}=E_{1}-E_{2}, \quad \overline{E_{2}}=E_{2}-E_{3}-E_{4},
$$

etc. So the tangency curve $D_{\text {tang }}$ between $\overline{\mathscr{H}}_{2}$ and $\overline{\mathscr{H}}_{3}$ satisfies

$$
\begin{aligned}
\mathcal{O}\left(D_{\text {tang }}\right)=f^{*} \mathcal{O}(6) \otimes & \mathcal{O} \\
& -2 E_{1}-3 E_{2}-2 E_{3}-3 E_{4}-E_{5} \\
& \left.-2 E_{6}-E_{7}-3 E_{8}-E_{9}-E_{10}-3 E_{11}\right) .
\end{aligned}
$$

Recalling that the tangency divisor $D_{\text {tang }}$ verifies $\mathcal{O}\left(D_{\text {tang }}\right)=T_{\overline{\mathcal{H}}_{2}}^{*} \otimes N_{\overline{\mathcal{H}_{3}}}$, we 
obtain

$$
\begin{gathered}
f^{*} \mathcal{O}\left(d\left(\mathscr{H}_{2}\right)+d\left(\mathscr{H}_{3}\right)+1\right) \otimes \mathcal{O}\left(-\sum_{i}^{11}\left(l\left(\mathscr{H}_{2}, p_{i}\right)-1+l\left(\mathcal{H}_{3}, p_{i}\right) \cdot E_{i}\right)\right. \\
=f^{*} \mathcal{O}(6) \otimes \\
\mathcal{O}\left(-2 E_{1}-3 E_{2}-2 E_{3}-3 E_{4}-E_{5}\right. \\
\left.-2 E_{6}-E_{7}-3 E_{8}-E_{9}-E_{10}-3 E_{11}\right) .
\end{gathered}
$$

From this isomorphism we obtain the following numerical equalities:

$$
\begin{aligned}
d\left(\mathscr{H}_{2}\right)+d\left(\mathscr{H}_{3}\right)=5, & \\
l\left(\mathcal{H}_{2}, p_{i}\right)+l\left(\mathcal{H}_{3}, p_{i}\right)=3 & \text { for } i=1,3,6, \\
l\left(\mathscr{H}_{2}, p_{i}\right)+l\left(\mathscr{H}_{3}, p_{i}\right)=4 & \text { for } i=2,4,8,11, \\
l\left(\mathscr{H}_{2}, p_{i}\right)+l\left(\mathscr{H}_{3}, p_{i}\right)=2 & \text { for } i=5,7,9,10 .
\end{aligned}
$$

Now we determine the numerical data of both foliations:

Proposition 2. For $\mathscr{H}_{2}$ we have

$$
\begin{aligned}
& d\left(\mathcal{H}_{2}\right)=2, \quad l\left(p_{1}\right)=1, \quad l\left(p_{2}\right)=2, \quad l\left(p_{3}\right)=1, \quad l\left(p_{4}\right)=2, \quad l\left(p_{5}\right)=1, \\
& l\left(p_{6}\right)=1, \quad l\left(p_{7}\right)=1, \quad l\left(p_{8}\right)=2, \quad l\left(p_{9}\right)=1, \quad l\left(p_{10}\right)=1, \quad l\left(p_{11}\right)=2,
\end{aligned}
$$

and for $\mathscr{H}_{3}$

$$
\begin{aligned}
& d\left(\mathcal{H}_{3}\right)=3, \quad l\left(p_{1}\right)=2, \quad l\left(p_{2}\right)=2, \quad l\left(p_{3}\right)=2, \quad l\left(p_{4}\right)=2, \quad l\left(p_{5}\right)=1, \\
& l\left(p_{6}\right)=2, \quad l\left(p_{7}\right)=1, \quad l\left(p_{8}\right)=2, \quad l\left(p_{9}\right)=1, \quad l\left(p_{10}\right)=1, \quad l\left(p_{11}\right)=2 .
\end{aligned}
$$

Proof. Both foliations, $\mathscr{H}_{2}$ and $\mathscr{H}_{3}$, admit as invariant algebraic curves the quintic $Q$ and the line $L_{\infty}$. This is sufficient to determine $\mathscr{H}_{2}$. If there exists $\mathcal{F}$ of degree 2 leaving $Q$ and $L_{\infty}$ invariant then the tangency locus of $\mathcal{F}$ and $\mathscr{H}_{2}$ would have degree 5 and would contain $Q$ and $L_{\infty}$, thus $\mathcal{F}$ and $\mathscr{H}_{2}$ should coincide. We determined $\mathscr{H}_{2}$ using the computer and after making its resolution, cf. Appendix B, we determined $l\left(p_{i}\right), i=1, \ldots, 11$, for $\mathscr{H}_{2}$.

From this data we obtain that

$$
T_{\overline{\mathcal{H}_{2}}}^{*}=\sigma^{*} \mathcal{O}(1) \otimes \mathcal{O}\left(-E_{2}-E_{4}-E_{8}-E_{11}\right) .
$$

Since the tangency locus of $\overline{\mathscr{H}}_{2}$ and $\overline{\mathscr{H}}_{3}$ is given by the formula

$$
\mathcal{O}\left(D_{\text {tang }}\right)=T_{\overline{\mathcal{H}_{2}}}^{*} \otimes N_{\overline{\mathcal{H}_{3}}}
$$

we obtain

$$
T_{\mathcal{H}_{3}}^{*}=\sigma^{*} \mathcal{O}(2) \otimes \mathcal{O}\left(-E_{1}-E_{2}-E_{3}-E_{4}-E_{6}-E_{8}-E_{11}\right) .
$$


Observe that this determine $l\left(p_{i}\right), i=1, \ldots, 11$, for $\mathscr{H}_{3}$. Therefore we can translate these conditions into an algebraic system of equations in the coefficients of the degree 3 vector fields. Solving this system with the help of a computer algebra system we can find the polynomial vector field of degree 3 with the numerical data prescribed (degrees and multiplicities along the resolution). In Appendix B we give in detail the reduction of singularities of $\mathscr{H}_{3}$ and also that of $\mathscr{H}_{2}$.

4.2.1. Canonical line bundles of $\overline{\mathscr{H}}_{2}$ and $\overline{\mathscr{H}}_{3}$. We describe the canonical line bundles of the modular foliations of $Y(5)$. These line bundles are explicit examples with numerical Kodaira dimension 1 and Kodaira dimension $-\infty$.

Remark, from the previous subsection, that

$$
T_{\mathcal{H}_{2}}^{* 2}=T_{\mathcal{H}_{3}}^{*}=-3 .
$$

From the Zariski decomposition $T_{\mathscr{H}_{2}}^{*}=P_{\mathscr{H}_{2}}+N_{\mathcal{H}_{2}}$ and $T_{\mathscr{H}_{3}}^{*}=P_{\mathcal{H}_{3}}+N_{\mathscr{H}_{3}}$ we conclude that $N_{\mathscr{H}_{2}}^{2}=N_{\mathscr{H}_{3}}^{2}=-3$. But the sporadicity property of the modular foliations imply that $N_{\mathscr{H}_{2}} \neq N_{\mathscr{H}_{3}}$. The rational coefficients of each component $N_{i}$ of the $N$-part of $T_{\mathcal{H}_{2}}^{*}$ and $T_{\mathcal{H}_{3}}^{*}$ are easily computed, provided we use the property of Zariski decomposition

$$
T_{\bar{H}}^{*} \cdot N_{i}=N \cdot N_{i},
$$

combined with the fact that each $N_{i}$ is $\overline{\mathscr{H}}_{2}$-invariant (McQuillan's theorem from Introduction) and Poincaré-Hopf theorem.

For this we need to know how many singularities exist over each $\bar{E}_{i}$ and their Poincaré-Hopf indices. This is provided by the study of the reduction of singularities in Appendix 2 (see Figures 17 and 18). We obtain

$$
N_{\overline{\mathcal{H}_{2}}}=\frac{3}{5} \overline{E_{1}}+\frac{1}{5} \overline{E_{2}}+\frac{2}{3} \overline{E_{3}}+\frac{1}{3} \bar{L}+\frac{1}{5} \overline{E_{5}}+\frac{2}{5} \overline{E_{6}}+\frac{1}{2} \overline{E_{7}}+\frac{1}{3} \overline{E_{9}}+\frac{1}{2} \overline{E_{1}}
$$

and

$$
N_{\overline{\mathscr{H}_{3}}}=\frac{1}{5} \overline{E_{1}}+\frac{2}{5} \overline{E_{2}}+\frac{1}{3} \overline{E_{3}}+\frac{2}{3} \bar{L}+\frac{1}{5} \overline{E_{5}}+\frac{3}{5} \overline{E_{6}}+\frac{1}{2} \overline{E_{7}}+\frac{1}{3} \overline{E_{9}}+\frac{1}{2} \overline{E_{10}}
$$

which satisfy $N_{\overline{\mathcal{H}}_{2}}^{2}=N_{\overline{\mathcal{H}}_{3}}^{2}=-3$.

\section{Projective models for modular foliations of $Y(5,(\sqrt{5}))$}

5.1. $Y(5, \sqrt{5})$ as a double covering of the plane. In this section we address Theorem 5 of the Introduction. The description of $Y(5, \sqrt{5})$ in [10] places it as an 
icosahedral-equivariant 2-fold ramified covering of the plane (blow up at 6 points). So in order to prove Theorem 5 , our first task is to show how to obtain $Y(5, \sqrt{5})$ from the plane just using blowing ups and blowing downs, which is not explicitly described in [10].

Known facts about $Y(5, \sqrt{5})$ from [10] are: i) $\mathbb{H}^{2} / \Gamma_{\sqrt{5}}$ is a smooth open surface, ii) its compactification $\overline{\mathbb{H}^{2} / \Gamma_{\sqrt{5}}}$ is done by adding six cusps. Each one of the 6 cusps of $Y(5,(\sqrt{5}))$ is desingularized as a cycle of two rational curves $A_{i} \cup B_{i}$, with intersection -3 .

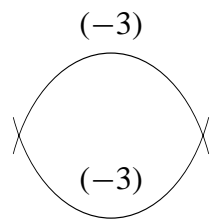

Figure 12. Cycles composed by two components on $Y(5,(\sqrt{5}))$.

Also it is known from [10] that the smooth rational curve which extends the diagonal in $\mathbb{H}^{2}$ to $Y(5,(\sqrt{5}))$, denoted by $C$, has self-intersection 2 . The action of $\mathrm{SL}_{2}(\mathcal{O}) / \Gamma_{\sqrt{5}}$ on $Y(5,(\sqrt{5}))$ carries $C$ to itself, and $C$ passes through each one of the six cycles $A_{i} \cap B_{i}$ at the nodal points (see Figure 13).

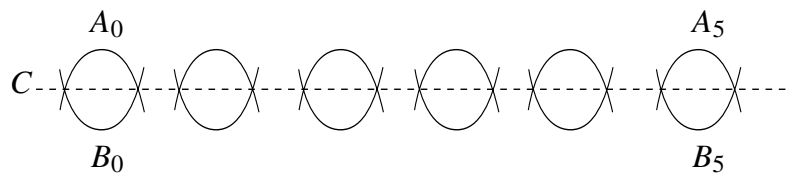

Figure 13. The resolution of cusps of $Y(5,(\sqrt{5}))$.

The extension of the involution $I: \mathscr{H}^{2} \circlearrowleft, I(x, y)=(y, x)$, is denoted also by $I: Y(5,(\sqrt{5}) \circlearrowleft$ and obviously $I(C)=C$. Consider the quotient $Y(5,(\sqrt{5})) / I$ and the 2 -fold covering

$$
p: Y(5,(\sqrt{5})) \rightarrow Y(5,(\sqrt{5})) / I
$$

ramified along $C$. It was also proven by Hirzebruch that $Y(5,(\sqrt{5})) / I$ is isomorphic to $\mathbb{P}^{2}$ blown up at the six points. These points are $v_{11}, \ldots, v_{16}$ given in Section 3 as associated to the vertices of the icosahedron. The ramification curve becomes in the plane (so after six blow downs) Klein's curve of degree 10, which is a rational curve, having singularities at $v_{11}, \ldots, v_{16}$ which are double cusps, invariant under the action of the Icosahedron in the plane. 


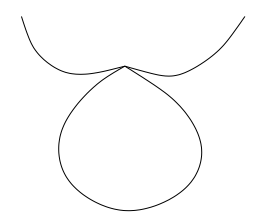

Figure 14. Double cusps of Klein degree 10 curve at the points $v_{i}, i=11, \ldots, 16$.

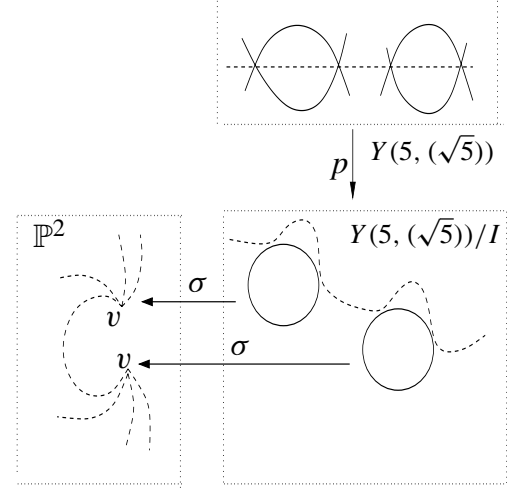

Figure 15. The 2-fold covering of $Y(5,(\sqrt{5}))$ over the blown up plane.

Remark that each cycle has self-intersection $\left(A_{i}+B_{i}\right)^{2}=-3-3+4=-2$ which is coherent with the fact that the cycle covers an exceptional line $E=\sigma^{-1}\left(v_{i}\right)$ (that is, $-2=2 \cdot E \cdot E$ ). Remark also that after 6 blow ups at $v_{i}$, the self-intersection of the degree 10 plane curve becomes equal to $10^{2}-6 \cdot 4^{2}=4\left(\right.$ since $\left.v\left(C, v_{i}\right)=4\right)$ and after covering it becomes equal to 2 .

5.2. $Y(5, \sqrt{5})$ as a modification of $\mathbb{P}^{2}$ and the minimal involution. The fact that the surface $Y(5,(\sqrt{5}))$ is a rational surface follows from the fact that there is a smooth rational curve with positive self-intersection, namely $C$ with $C^{2}=2$. Our aim now is to obtain it from the plane by means of blowing ups and blowing downs.

Consider in $\mathbb{P}^{2}$ the six conics $C_{i_{1}, \ldots, i_{5}}$ passing through 5 among the 6 vertices $v_{11}, \ldots, v_{16}$. After the blow ups, these conics becomes $(-1)$-curves $\bar{C}_{i_{1}, \ldots, i_{5}}$ in $Y(5,(\sqrt{5})) / I$. We claim that $\bar{C}_{i_{1}, \ldots, i_{5}}$ does not intersect the transformed curve of $C$. In fact $C_{i_{1}, \ldots, i_{5}}$ and $C$ intersect at 5 among the 6 vertices $v_{i j}$ at which $v\left(C, v_{i j}\right)=4$. Thus the local intersection at each of these points is at least 4 and it is greater than 4 if, and only if, $\bar{C}_{i_{1}, \ldots, i_{5}}$ intersects the strict transform of $C$. Since we have 5 such points and $C \cdot C_{i_{1}, \ldots, i_{5}}=20$, the claim follows. 
Therefore, taking the 2-fold covering, there are 12 pairwise disjoint (-1)-curves, which are the pre-images of the curves $\bar{C}_{i_{1}, \ldots, i_{5}}$. Each curve $A_{i}$ and $B_{i}$ of the cycles intersects 5 of these $12(-1)$-curves (see Figure 16)

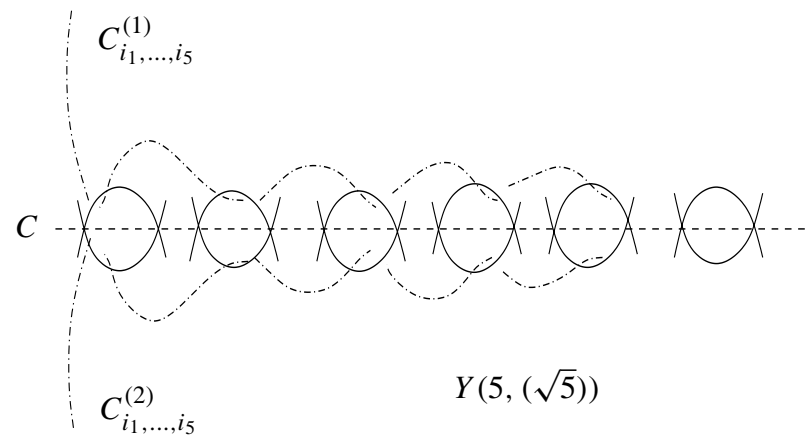

Figure 16. Curves $C_{i_{1}, \ldots, i_{5}}^{(1)}$ and $C_{i_{1}, \ldots, i_{5}}^{(2)}$ are pre-images of $\bar{C}_{i_{1}, \ldots, i_{5}}$.

Now we consider the map $h: Y(5,(\sqrt{5})) \rightarrow W$, which corresponds to the blow down of all the 12 exceptional lines described in $Y(5,(\sqrt{5}))$.

Then it is clear that the Euler characteristic of $W$ is $e(W)=e(Y(5,(\sqrt{5}))-12$. It is proven in [10] that $e(Y(5,(\sqrt{5}))=16$. So the rational surface $W$ with $e(W)=4$ is a Hirzebruch surface $\Sigma_{n}$. Also we see that the transformed curves of $A_{i}$ and $B_{i}$ in $S$ have self-intersection $-3+5=2$. Now, using that these rational curves have self-intersection 2 in the surface $W=\Sigma_{n}$, see [9], we can show that $W=\mathbb{P}_{\mathbb{C}}^{1} \times \mathbb{P}_{\mathbb{C}}^{1}$.

5.3. Numerical data of the foliations. In order to find the numerical data of the modular foliations in $W=\mathbb{P}_{\mathbb{C}}^{1} \times \mathbb{P}_{\mathbb{C}}^{1}$, let us apply the tangency formula to the image of $C$ in $W=\mathbb{P}_{\mathbb{C}}^{1} \times \mathbb{P}_{\mathbb{C}}^{1}$, whose self-intersection 2 has not changed by the map $h: Y(5,(\sqrt{5})) \rightarrow W$. We have

$$
T_{\mathcal{F}}^{*} \cdot C=\operatorname{tang}(C, \mathcal{F})-C^{2}=12-2
$$

since the twelve reduced singularities along the cycles $A_{i} \cup B_{i}$ belong to $C$. Then $d_{1}(\mathcal{F})+d_{2}(\mathcal{F})=10\left(\right.$ also $\left.d_{1}(\mathcal{G})+d_{2}(\mathcal{G})=10\right)$. The tangency curve of the pair of modular foliations in $\mathbb{P}_{\mathbb{C}}^{1} \times \mathbb{P}_{\mathbb{C}}^{1}$ is then composed by 12 curves of bi-degrees $(1,1)$, images of the components of cycles by $h$. If we denote again by $\mathcal{F}$ and $g$ the foliations in $\mathbb{P}_{\mathbb{C}}^{1} \times \mathbb{P}_{\mathbb{C}}^{1}$ then the tangency along these curves produces the relations

$$
d_{1}(\mathcal{F})+d_{2}(\mathcal{G})+2=d_{2}(\mathcal{F})+d_{1}(\mathcal{G})+2=12 .
$$

Let us now determine the Milnor numbers of the 12 dicritical singularities $r_{i}$, introduced by the blowing downs. For computing this, we use Darboux's theorem in 
$\mathbb{P}_{\mathbb{C}}^{1} \times \mathbb{P}_{\mathbb{C}}^{1}$, taking into consideration the twelve saddle singularities along $C$ :

$$
12+\sum_{i=1}^{12} \mu\left(r_{i}\right)-4=T_{\mathcal{F}}^{*} \cdot N_{\mathcal{F}} .
$$

Then

$$
\begin{aligned}
\sum_{i=1}^{12} \mu\left(r_{i}, \mathcal{F}\right) & =2 \cdot\left(d_{1}(\mathcal{F}) \cdot d_{2}(\mathcal{F})+d_{1}(\mathcal{F})+d_{2}(\mathcal{F})\right)-8 \\
& =2 d_{1}(\mathcal{F}) \cdot d_{2}(\mathcal{F})+12
\end{aligned}
$$

and so, for each $r_{i}$,

$$
\mu\left(r_{i}, \mathcal{F}\right)=\frac{d_{1}(\mathcal{F}) \cdot d_{2}(\mathcal{F})}{6}+1 .
$$

The unique solution of this equation for (non-zero) bi-degrees (whose sum we know is 10 ) is $d_{1}(\mathcal{F})=4, d_{2}(\mathcal{F})=6$ (or vice-versa) and $\mu\left(r_{i}, \mathcal{F}\right)=5$. After we know the Milnor numbers, the invariants $l\left(r_{i}, \mathcal{F}\right)$ are easily computed: they are equal to 3 for all $i=1, \ldots, 12$.

Now, by an elementary transformation $E: \mathbb{P}_{\mathbb{C}}^{1} \times \mathbb{P}_{\mathbb{C}}^{1}-->\mathbb{P}^{2}$ (cf. Section 3.4.1) we get foliations in the plane with the same degrees. As remarked in that section, these transformations are defined by blowing up a point $p$ and subsequent contraction of the transformed curves of horizontal and vertical lines, denoted $\bar{H}$ and $\bar{V}$. For instance, if we blow up a regular point $p$ for the foliations, we get foliations in the plane whose degrees are both equal to 12 . On the other hand, if we choose the elementary transformation which blows up one dicritical point $p=r_{i}$, we get degree 9 for both modular foliations. In this model, the algebraic curves invariant by the modular foliations are 5 straight lines and 7 conics. The lines are images in the plane of the $(1,1)$-curves passing through $p=r_{i}$, whose self-intersection decreases by one by the blow up at $p$ and which are not affected by the contraction of $\bar{H}$ and $\bar{V}$. The conics correspond to the $(1,1)$-curves not affected by the blow up at $p$ but whose self-intersection 2 is increased by 2 under the contractions of $\bar{H}$ and $\bar{V}$.

At last, a remark on the canonical line bundles is in place. If $\overline{\mathcal{F}}$ denotes a reduction of singularities of the modular foliation in $\mathbb{P}_{\mathbb{C}}^{1} \times \mathbb{P}_{\mathbb{C}}^{1}$, obtained from 12 blow ups at dicritical points, then $T_{\mathcal{F}}^{*}$ is nef (the $N$-part is empty) and

$$
T_{\overline{\mathcal{F}}}^{*}=\mathcal{O}(4 H+6 V) \otimes \mathcal{O}\left(-\sum_{i=1}^{12} 2 E_{i}\right)
$$

gives $T_{\overline{\mathcal{F}}}^{*} \cdot T_{\overline{\mathcal{F}}}^{*}=0$. Also $T_{\bar{g}}^{*} \cdot T_{\bar{g}}^{*}=0$ holds for the companion foliation, since $T_{\bar{g}}^{*}=\mathcal{O}(6 H+4 V) \otimes \mathcal{O}\left(-\sum_{i=1}^{12} 2 E_{i}\right)$. 


\section{A. Factorization of the Cremonian involution}

In this section we construct the involution $\mathcal{T}$ in the plane associated to the involution of $Y(5,(2))$ (which sends $\mathscr{H}_{5}$ to $\mathscr{H}_{9}$ in Theorem 2 ) as a composition of three quadratic transformations of the plane:

$$
\mathcal{T}=Q_{3} \circ Q_{2} \circ Q_{1} .
$$

The transformation $\mathcal{T}$ is a degree five Cremonian transformation with six indetermination points and is described by Godeaux [8] as a degenerated case of a Geiser involution (in general a degree eight involution with seven indetermination points). In this particular case, the 2-net of rational curves defining $\mathcal{T}$ is composed by degree five curves with double points at the six indetermination points. According to [8], in this case, there is a non-empty fixed part of degree 3 in the 2-net of curves of degree 8 defining the general Geiser involution.

Moreover, in this section we construct birational modifications of $\mathscr{H}_{5}$, denoted $\mathscr{H}_{k}, k=6,8,9$ of degrees $6,8,9$, respectively, given as follows: $\mathscr{H}_{6}=\left(Q_{1}^{-1}\right)^{*}\left(\mathscr{H}_{5}\right)$, $\mathscr{H}_{8}=\left(\left(Q_{2} \circ Q_{1}\right)^{-1}\right)^{*}\left(\mathscr{H}_{5}\right)$ and $\mathscr{H}_{9}=\left(\left(Q_{3} \circ Q_{2} \circ Q_{1}\right)^{-1}\right)^{*}\left(\mathscr{H}_{5}\right)$. With this factorization process we are also led to a better understanding of the effect of $\mathcal{T}=\mathcal{T}_{5}$ on the foliations and on the configuration of lines.

The standard quadratic transformation of the plane, $Q: \mathbb{P}^{2}-->\mathbb{P}^{2}$, is given in homogeneous coordinates by $Q\left(x_{0}: x_{1}: x_{2}\right)=\left(x_{1} \cdot x_{2}: x_{0} \cdot x_{2}: x_{0} \cdot x_{1}\right)$. It factorizes as the blow up at $e_{1}:=(1: 0: 0), e_{2}:=(0: 1: 0), e_{3}:=(0: 0: 1)$, with $E_{i}=\sigma_{i}^{-1}\left(e_{i}\right)$, followed by the blow downs of the strict transforms $\overline{L_{k}}$ of the three lines $L_{k}:=e_{i} \cdot e_{j}$ to points $q_{k}, k=1,2,3$. The strict transforms of $E_{i}$ are three lines $E_{i}^{\prime} \subset \mathbb{P}^{2}$ connecting two points among $q_{k}$.

Lemma 1. Let $Q: \mathbb{P}^{2}-->\mathbb{P}^{2}$ be the standard quadratic transformation (keeping the previous notations). If $C$ is a degree $d=d(C)$ curve, then the degree of the strict transform $Q(C)$ is $2 \cdot d(C)-\sum_{i=1}^{3} v_{e_{i}}(C)$, where $v_{e_{i}}(C)$ is the algebraic multiplicity. Moreover

$$
v_{q_{k}}(Q(C))=d(C)-v_{e_{i}}(C)-v_{e_{j}}(C), \quad i \neq j \neq k \in\{1,2,3\} .
$$

If $\mathcal{H}$ is a foliation of degree $d=d(\mathscr{H})$, then the degree of the foliation $Q_{*}(\mathcal{H})$ (with isolated singularities) is equal to $2 \cdot d(\mathcal{H})+2-\sum_{i=1}^{3} l\left(e_{i}, \mathcal{H}\right)$. Furthermore

$$
l\left(q_{k}, Q_{*}(\mathcal{H})\right)=d(\mathscr{H})+2-l\left(e_{i}, \mathscr{H}\right)-l\left(e_{j}, \mathscr{H}\right), \quad i \neq j \neq k \in\{1,2,3\} .
$$

Proof. The assertion on curves in this lemma is well known from classical books on algebraic curves.

The assertion on the degrees of foliations can be proven if we remark that by definition, $d\left(Q_{*}(\mathscr{H})\right)$ is the sum of tangencies with a generic straight line $L$; but $L$ 
is the strict transform by $Q$ of a conic $C$ passing through $e_{1}, e_{2}$ and $e_{3}$. So the proof follows easily from the formula for the variation of order of tangencies under blow ups (at the end of Section 2.2.3) and from the formula

$$
\operatorname{tang}(C, \mathscr{H})=N_{\mathscr{H}} \cdot C-\chi(C)=2 \cdot d(\mathscr{H})+2 .
$$

As explained in Section 2.2.3, the computation of $l\left(q_{k}, Q_{*}(\mathscr{H})\right)$ depends on the sum of tangencies along the exceptional line $\overline{L_{k}}$ (if it is not invariant by the foliation) or on the sum of Poincaré-Hopf indices along $\overline{L_{k}}$ and is easily done.

Let us start the definition of $\mathcal{T}=Q_{3} \circ Q_{2} \circ Q_{1}$.

Definition of $Q_{1}$. By a linear transformation of the plane we can put three vertices as

$$
e_{1}=v_{11}, \quad e_{2}=v_{13}, \quad e_{3}=v_{15}
$$

and take for $Q_{1}$ the standard transformation based on these points. So the strict transforms of $L_{B_{2}}=\overline{v_{11} v_{13}}, L_{B_{3}}=\overline{v_{13} v_{15}}$ and $L_{C_{0}}=\overline{v_{11} v_{15}}$ by the blow ups done by $Q_{1}$ will be contracted, producing again the projective plane.

By Lemma 1, the transforms of $L_{B_{0}}=\overline{v_{14} v_{16}}, L_{A_{2}}=\overline{v_{12} v_{16}}$ and $L_{A_{3}}=\overline{v_{12} v_{14}}$ (cf. Section 3) under the Cremonian transformation are conics passing through the points introduced by contraction of the strict transforms of $L_{B_{2}}, L_{B_{3}}$ and $L_{C_{0}}$, which we denote $q_{B_{2}}, q_{B_{3}}$ and $q_{C_{0}}$, respectively. All other lines $L_{A_{i}}, L_{B_{i}}, L_{C_{i}}$ in the configuration are transformed into straight lines, because each one has received one blow up at one point from $\left\{v_{11}, v_{13}, v_{15}\right\}$.

Let $\mathscr{H}_{5}^{\prime}$ denote the transformed foliation $\left(Q_{1}^{-1}\right)^{*}\left(\mathscr{H}_{5}\right)$ (with isolated singularities). By Lemma $1, d\left(\mathcal{H}_{5}^{\prime}\right)=2 \cdot 5+2-3 \cdot 2=6$ and its singular set is the following:

i. $\mathcal{H}_{5}^{\prime}$ has singularities at the images of $o_{1}, o_{2}, o_{5}, o_{9}, v_{12}, v_{14}$ and $v_{16}$ by $Q_{1}$ that are isomorphic to those of $\mathscr{H}_{5}$, that is, are radial points.

ii. There are degenerate singularities of $\mathscr{H}_{5}^{\prime}$ at $q_{B_{2}}, q_{B_{3}}$ and $q_{C_{0}}$. The reduction of singularities of $q_{B_{2}}$ is as follows: one blow up produces an invariant exceptional line $E$ with two radial points, isomorphic to the radial points $o_{6}$ and $o_{4}$ (which need to be blown up again) and two more reduced singularities isomorphic to the singularities $L_{A_{2}} \cap L_{B_{2}}$ and $L_{C_{2}} \cap L_{B_{2}}$ of $\mathcal{F}$. The picture is like that of Figure 8 in Section 3.4 (see page 266).

In order to compute the algebraic multiplicity of $\mathcal{H}_{5}^{\prime}$ at $q_{B_{2}}, q_{B_{3}}$ and $q_{C_{0}}$, we use the known formula

$$
\sum_{p \in E} \mu\left(\sigma^{*}\left(\mathscr{H}_{5}^{\prime}\right), p\right)=\mu_{q}-m_{q}\left(\mathcal{H}_{5}^{\prime}\right) \cdot\left(m_{q}\left(\mathscr{H}_{5}^{\prime}\right)-1\right)+1,
$$

which gives

$$
4=\sum_{p \in E} \mu_{p}\left(\sigma^{*}\left(\mathcal{H}_{5}^{\prime}\right)\right)=\mu_{q_{B_{2}}}\left(\mathcal{H}_{5}^{\prime}\right)-m_{q_{B_{2}}}\left(\mathcal{H}_{5}^{\prime}\right) \cdot\left(m_{q_{B_{2}}}\left(\mathcal{H}_{5}^{\prime}\right)-1\right)+1
$$


and, summing up for the three points,

$$
\begin{aligned}
m_{q_{B_{2}}} & \cdot\left(m_{q_{B_{2}}}-1\right)+m_{q_{B_{3}}} \cdot\left(m_{q_{B_{3}}}-1\right)+m_{q_{C_{0}}} \cdot\left(m_{q_{C_{0}}}-1\right)+9 \\
& =\mu_{q_{B_{2}}}\left(\mathcal{H}_{5}^{\prime}\right)+\mu_{q_{B_{3}}}\left(\mathcal{H}_{5}^{\prime}\right)+\mu_{q_{C_{0}}}\left(\mathcal{H}_{5}^{\prime}\right) \\
& =\left[6^{2}+6+1-\left(5^{2}+5+1-3-3 \cdot 4\right)\right] \\
& =27
\end{aligned}
$$

from the homogeneity in the definition of $q_{B_{2}}, q_{B_{3}}, q_{C_{0}}$ we obtain

$$
m_{q_{B_{2}}}=m_{q_{B_{3}}}=m_{q_{C_{0}}}=3 .
$$

At this point we can verify directly that the numerical Kodaira dimension of $\mathscr{H}_{5}^{\prime}$ is one, exemplifying the birational invariance of this concept; we have

$$
\begin{aligned}
\left(d\left(\mathcal{H}_{5}^{\prime}\right)-1\right)^{2}= & \left(m\left(q_{B_{2}}, \mathscr{H}_{5}^{\prime}\right)-1\right)^{2}+\left(m\left(q_{B_{3}}, \mathcal{H}_{5}^{\prime}\right)-1\right)^{2} \\
& +\left(m\left(q_{C_{0}}, \mathscr{H}_{5}^{\prime}\right)-1\right)^{2}+\sum_{p \neq q_{B_{2}}, q_{B_{3}}, q_{C_{0}}} m\left(p, \mathcal{H}_{5}^{\prime}\right)^{2},
\end{aligned}
$$

where in

$$
\sum_{p \neq q_{B_{2}}, q_{B_{3}}, q_{C_{0}}} m\left(p, \mathcal{H}_{5}^{\prime}\right)^{2}
$$

we include the contribution of the blow ups of radial points along the exceptional introduced by blow up of $q_{B_{2}}, q_{B_{3}}, q_{C_{0}}$, that is, we have the equality $25=3 \cdot 4+13$.

We conclude that $\mathscr{H}_{5}^{\prime}$ is a degree 6 modular foliation, denoted $\mathscr{H}_{6}=\left(Q_{1}\right)_{*}\left(\mathscr{H}_{5}\right)$.

Definition of $\boldsymbol{Q}_{\mathbf{2}}$. Now consider the points $Q_{1}\left(v_{12}\right), Q_{1}\left(v_{14}\right), Q_{1}\left(v_{16}\right)$. They are not collinear and we can take a linear transformation putting

$$
\begin{aligned}
& e_{1}=(1: 0: 0)=Q_{1}\left(v_{12}\right), \\
& e_{2}=(0: 1: 0)=Q_{1}\left(v_{14}\right), \\
& e_{3}=(0: 0: 1)=Q_{1}\left(v_{16}\right),
\end{aligned}
$$

and we define $Q_{2}$ as the standard quadratic transformation with base points at these points. Since $\mathscr{H}_{6}$ has $l_{e_{i}}\left(\mathscr{H}_{6}\right)=2$, we obtain from Lemma 1

$$
\begin{aligned}
d\left(\left(Q_{2}\right)_{*} \mathcal{H}_{6}\right) & =2 \cdot 6+2-3 \cdot 2=8, \\
l_{q_{k}}\left(\left(Q_{2}\right)_{*} \mathscr{H}_{6}\right) & =6+2-2 \cdot 2=4 .
\end{aligned}
$$

Remark that the lines $L_{k}:=\overline{e_{i} e_{j}}$ are not $\mathscr{H}_{6}$-invariant. Since $e_{i}$ and $e_{j}$ are radial points for $\mathscr{H}_{6}, \operatorname{tang}\left(L_{k}, e_{i}\right)+\operatorname{tang}\left(L_{k}, e_{i}\right)=4$ and the points $q_{k}$ introduced by the blow downs of (the transforms of) $L_{k}$ are dicritical and we have $l_{q_{k}}\left(\left(Q_{2}\right)_{*} \mathscr{H}_{6}\right)=4$. So we have obtained that $\left(Q_{2}\right)_{*} \mathscr{H}_{6}=\left(Q_{2} \circ Q_{1}\right)_{*} \mathscr{H}_{5}$ is a model $\mathscr{H}_{8}$ of degree 8 . 
Definition of $Q_{3}$. In order to complete the construction of the Cremonian transformation $\mathcal{T}=Q_{3} \circ Q_{2} \circ Q_{1}$, let us define $Q_{3}$. For doing this, take a linear transformation of the plane such that

$$
\begin{aligned}
& e_{1}=(1: 0: 0)=Q_{2}\left(Q_{1}\left(v_{11}\right)\right), \\
& e_{2}=(0: 1: 0)=Q_{2}\left(Q_{1}\left(v_{13}\right)\right), \\
& e_{3}=(0: 0: 1)=Q_{2}\left(Q_{1}\left(v_{15}\right)\right) .
\end{aligned}
$$

Now again by Lemma 1 ,

$$
\begin{aligned}
d\left(\left(Q_{3}\right)_{*} \mathscr{H}_{8}\right) & =2 \cdot 8+2-3 \cdot 3=9 \\
l_{q_{k}}\left(\left(Q_{3}\right)_{*} \mathscr{H}_{8}\right) & =8+2-2 \cdot 3=4
\end{aligned}
$$

and $q_{k}$ are dicritical points eliminable by one blow up, since $Q_{3}$ has blown up all singular points of $\mathscr{H}_{8}$ that needed more that one blow up in its reduction. In this way we have obtained the model of degree 9 in the pair $\left(\mathscr{H}_{5}, \mathscr{H}_{9}\right)$ as $\mathscr{H}_{9}=\mathcal{T}_{*}\left(\mathscr{H}_{5}\right)$.

The composition $\mathcal{T}=Q_{3} \circ Q_{2} \circ Q_{1}$ of three quadratic transformations has degree five as a birational transformation (the degree of the composition is not eight, because the base points of the quadratic transformation in the composition are not disjoint). In order to see this, consider the image of the 2-dimensional linear system of curves of degree 5 passing doubly through $v_{11}, \ldots, v_{16}$. If $C$ denotes one of these curves, the degrees of its strict transforms are computed, by means of the previous lemma as

$$
d\left(Q_{1}(C)\right)=2 \cdot 5-3 \cdot 2=4, \quad v_{q_{k}}\left(Q_{1}(C)\right)=1
$$

and

$$
d\left(Q_{2}\left(Q_{1}(C)\right)=2 \cdot d\left(Q_{1}(C)\right)-3 \cdot 2=2 .\right.
$$

Finally $d\left(Q_{3}\left(Q_{2}\left(Q_{1}(C)\right)\right)=2 \cdot d\left(Q_{2}\left(Q_{1}(C)\right)-3 \cdot 1=2 \cdot 2-3=1\right.\right.$ which gives a 2-dimensional system of lines, that is $\mathbb{P}^{2}$. The birational transformation $\mathcal{T}: \mathbb{P}^{2}-->\mathbb{P}^{2}=N^{\prime}$ can be given as $T\left(x_{0}: x_{1}: x_{2}\right)=\left(P_{0}: P_{1}: P_{2}\right)$ where $P_{0}, P_{1}, P_{2}$ is a basis of the $\mathbb{C}$-vector space of polynomials of degree 5 vanishing with order two at the five points $v_{11}, \ldots, v_{16}$.

At last, let us explain how the transformation $\mathcal{T}=Q_{1} \circ Q_{2} \circ Q_{3}$ does preserve the configuration of 15 lines $L_{A_{i}}, L_{B_{i}}, L_{C_{i}}$, although this is not the case for any of the quadratic transformations $Q_{i}, i=1,2,3$.

For showing this, let us divide the set of 15 lines into three subsets; a) lines that do not pass through $v_{11}$, neither by $v_{13}$, nor $v_{15} ;$ b) lines that pass through exactly one point in $\left\{v_{11}, v_{13}, v_{15}\right\}$; and c) lines that pass through a pair of points from $\left\{v_{11}, v_{13}, v_{15}\right\}$.

In case a), for fixing ideas, take the line $L_{B_{0}}=\overline{v_{14} v_{16}}$. The strict transform $Q_{1}\left(B_{0}\right)$ is a conic. Since $B_{0}$ does not contain $v_{12}$, the transformation $Q_{2}$ operates on $Q_{1}\left(B_{0}\right)$ by means of two blow ups at $Q_{1}\left(v_{14}\right), Q_{1}\left(v_{16}\right)$ and so $d\left(Q_{2}\left(Q_{1}\left(C_{0}\right)\right)\right)=$ 
$2 \cdot 2-2=2$, and $Q_{2}\left(Q_{1}\left(B_{0}\right)\right)$ also is a conic. Now since $Q_{1}\left(B_{0}\right)$ contains $q_{C_{0}}, q_{B_{3}}$, $q_{B_{2}}$, we conclude that $d\left(Q_{3}\left(Q_{2}\left(Q_{1}\left(B_{0}\right)\right)\right)\right)=2 \cdot 2-3=1$ and we obtain a line as desired.

In case $\mathrm{b}$ ), if a line in the configuration contains just one point from $\left\{v_{11}, v_{13}, v_{15}\right\}$, for fixing ideas let us suppose this point is $v_{11}$. Then $Q_{1}(L)$ is also a line, passing through just one of the points $q_{C_{0}}, q_{B_{3}}, q_{B_{2}}$, namely by $q_{B_{3}}$, because $L_{B_{3}}=\overline{v_{13} v_{15}}$.

But any line in the configuration of 15 lines must contain 2 vertices $v_{i j}$. Now $v_{11} \in$ $L, v_{13}, v_{15} \notin L$, hence $L$ contains exactly one point from $\left\{v_{12}, v_{14}, v_{16}\right\}$. So when applying $Q_{2}$ exactly one point of $Q_{1}(L)$ (a line) is blown up and $d\left(Q_{2}\left(Q_{1}(L)\right)\right)=$ $2 \cdot 1-1=1$. When applying $Q_{3}$ we blow up $Q_{2}\left(p_{B_{3}}\right)$; so $d\left(Q_{3}\left(Q_{2}\left(Q_{1}(L)\right)\right)\right)=$ $2 \cdot 1-1=1$ and again we have a line.

Finally, in the case c), if a line in the configuration contains a pair of points from $\left\{v_{11}, v_{13}, v_{15}\right\}$ it does not contains the third one (they are not collinear). But then $L$ is blown down by $Q_{1}$ and re-introduced as one of the three lines created by $Q_{3}$.

\section{B. Reduction of singularities of the plane models of $Y(5)$}

Here we give a detailed reduction of singularities for the modular foliations in the plane associated to $Y(5)$, given in Proposition 2. Figures 17 and 18 present all the reduction processes, composed each by eleven blowing ups $f: Y(5) \rightarrow \mathbb{P}^{2}$. Remark that the reduced singularities which are not at the corners of the exceptional divisors are denoted respectively by $q_{i}, q_{L}$ and $r_{i}$ in these figures.

B.1. Resolution of $\mathscr{H}_{2}$. We begin with the reduction of singularities of $\mathscr{H}_{2}$ at infinity in the plane. The foliation $\mathscr{H}_{2}$ is induced in affine coordinates $(x, y)$ of the projective plane by

$$
\Omega=\left(80 y-60 x y-80 x^{2}\right) d x-\left(y+32 x-36 x^{2}\right) d y=0 .
$$

In the chart $(u, v)=\left(\frac{1}{x}, \frac{y}{x}\right)$ there is a reduced singularity at $q_{L}:=\left(0,-\frac{10}{3}\right)=(u, v)$ (with Camacho-Sad index $-\frac{3}{2}$ relative to the line at infinity), as can be easily verified. The foliation $\mathscr{H}_{2}$ is induced at the point at infinity $p_{1}:=(0,0)=(w, s)=\left(\frac{x}{y}, \frac{1}{y}\right)$ by

$$
\Omega(w, s)=\left(80 s^{2}-60 w s-80 w^{2} s\right) d w+\left(s-48 w s+24 w^{2}+80 w^{3}\right) d s=0,
$$

where $s=0$ is an affine equation of the $\mathscr{H}_{2}$-invariant line at infinity. The blowing up $\sigma_{1}$ at $p_{1}$ is written in local charts as

$$
\sigma_{1}\left(x_{1}, t_{1}\right)=\left(x_{1}, x_{1} t_{1}\right)=(w, s), \quad \sigma_{1}\left(u_{1}, y_{1}\right)=\left(u_{1} y_{1}, y_{1}\right)=(w, s),
$$

and

$\sigma_{1}^{*} \Omega(w, s)=x_{1} \cdot\left[\left(-36 x_{1} t_{1}+t_{1}^{2}+32 x_{1} t_{1}^{2}\right) d x_{1}+\left(x_{1} t_{1}+24 x_{1}^{2}-48 x_{1}^{2} t_{1}+80 x_{1}^{3}\right) d t_{1}\right]$, that is, $l\left(\mathscr{H}_{2}, p_{1}\right)=1$. 


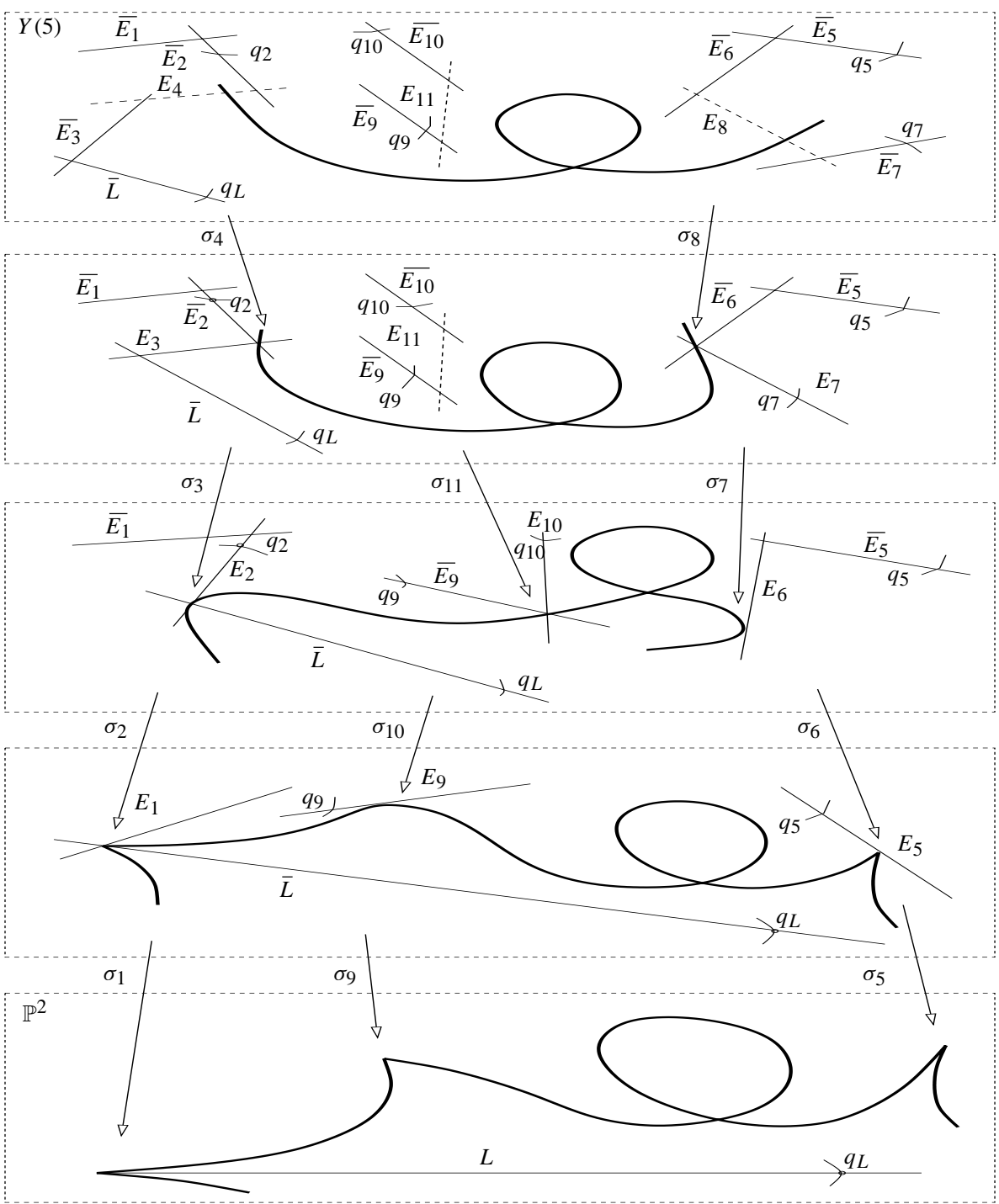

Figure 17. Resolution of $\mathscr{H}_{2}$. 


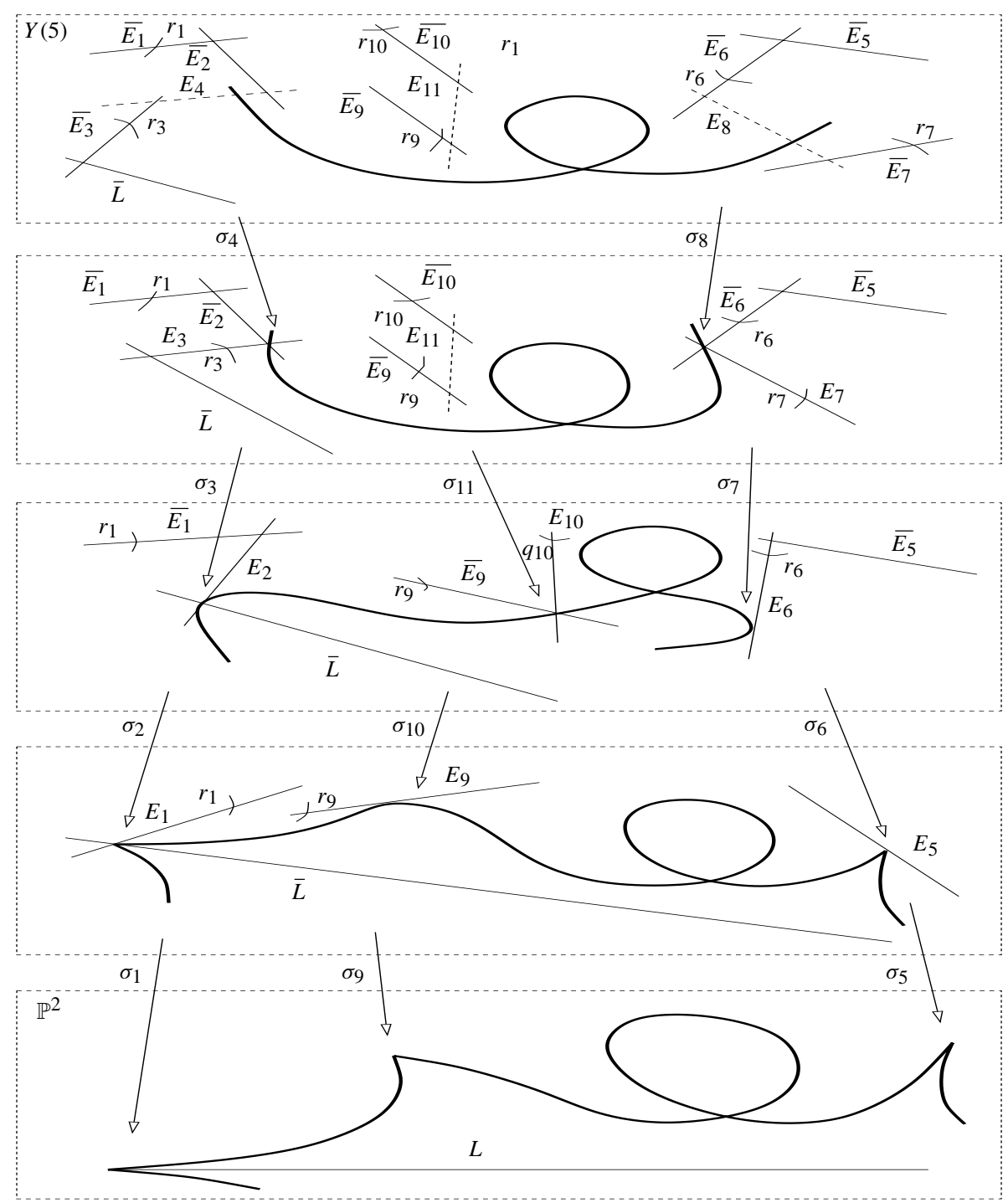

Figure 18. Resolution of $\mathscr{H}_{3}$. 
The unique singularity of the transformed foliation along $E_{1}:=\sigma_{1}^{-1}\left(p_{1}\right)$ is at $(0,0)=\left(x_{1}, t_{1}\right)$, as is easily verified. The blowing up $\sigma_{2}$ at $p_{2}:=(0,0)=\left(x_{1}, t_{1}\right)$ is written in local charts as

$$
\sigma_{2}\left(x_{2}, t_{2}\right)=\left(x_{2}, x_{2} t_{2}\right)=\left(x_{1}, y_{1}\right), \quad \sigma_{2}\left(u_{2}, y_{2}\right)=\left(u_{2} y_{2}, y_{2}\right)=\left(x_{1}, y_{1}\right),
$$

and

$$
\begin{gathered}
\sigma_{2}^{*}\left[\left(-36 x_{1} t_{1}+t_{1}^{2}+32 x_{1} t_{1}^{2}\right) d x_{1}+\left(x_{1} t_{1}+24 x_{1}^{2}+80 x_{1}^{3}-48 x_{1}^{2} t_{1}\right) d t_{1}\right] \\
=x_{2}^{2} \cdot\left[\left(-12 t_{2}-16 x_{2} t_{2}^{2}+80 x_{2} t_{2}+2 t_{2}^{2}\right) d x_{2}\right. \\
\left.+\left(24 x_{2}+t_{2} x_{2}+80 x_{2}^{2}-48 x_{2}^{2} t_{2}\right) d t_{2}\right],
\end{gathered}
$$

that is, $l\left(\mathscr{H}_{2}, p_{2}\right)=2$.

The non-reduced singularity of the transformed foliation along $E_{2}$ is at $(0,0)=$ $\left(x_{2}, t_{2}\right)$. There are also two reduced singularities, one at $q_{2}:=(0,6)=\left(x_{2}, t_{2}\right)$ (with Camacho-Sad index $-\frac{5}{2}$ relative to $\left.E_{2}\right)$ and the other is at infinity $(0,0)=$ $\left(u_{2}, y_{2}\right)=\overline{E_{1}} \cap E_{2}$ (with Camacho-Sad index $-\frac{1}{2}$ relative to $\left.E_{2}\right)$.

The blowing up $\sigma_{3}$ at $p_{3}:=(0,0)=\left(x_{2}, t_{2}\right)$ is written in local charts as

$$
\sigma_{3}\left(x_{3}, t_{3}\right)=\left(x_{3}, x_{3} t_{3}\right)=\left(x_{2}, y_{2}\right), \quad \sigma_{3}\left(u_{3}, y_{3}\right)=\left(u_{3} y_{3}, y_{3}\right)=\left(x_{2}, y_{2}\right),
$$

and

$$
\begin{gathered}
\sigma_{3}^{*}\left[\left(-12 t_{2}-16 x_{2} t_{2}^{2}+80 x_{2} t_{2}+2 t_{2}^{2}\right) d x_{2}+\left(24 x_{2}+t_{2} x_{2}+80 x_{2}^{2}-48 x_{2}^{2} t_{2}\right) d t_{2}\right] \\
=x_{3} \cdot\left[\left(24 x_{3}+80 x_{3}^{2}-47 x_{3}^{2} t_{3}+\right) d t_{3}\right. \\
\left.\quad+\left(12 t_{3}+160 x_{3} t_{3}+3 x_{3} t_{3}^{2}-64 x_{3}^{2} t_{3}^{2}\right) d x_{3}\right]
\end{gathered}
$$

that is, $l\left(\mathscr{H}_{2}, p_{3}\right)=1$.

There is a reduced singularity at $(0,0)=\left(x_{3}, t_{3}\right)$ which is the crossing point $E_{3} \cap \bar{L}$ (with Camacho-Sad index -2 relative to $E_{3}$ ). The point at infinity $p_{4}:=$ $(0,0)=\left(u_{3}, y_{3}\right), p_{4}=E_{3} \cap \overline{E_{2}}$, is a non-reduced singularity, where the transformed foliation is induced by

$$
\left(12 u_{3}+3 u_{3} y_{3}+160 u_{3}^{2} y_{3}-64 u_{3}^{2} y_{3}^{2}\right) d y_{3}+\left(-12 y_{3}+2 y_{3}^{2}+80 u_{3} y_{3}^{2}-16 u_{3} y_{3}^{3}\right) d u_{3}=0,
$$

which clearly is a dicritical point of radial type, that is, $l\left(\mathscr{H}, p_{4}\right)=2$.

Let us now consider the reduction of $\mathscr{H}_{2}$ at $p_{5}:=(0,0)=(x, y)$ in the projective plane. The blowing up $\sigma_{5}$ at $p_{5}$ is written in local charts as

$$
\sigma_{5}\left(x_{5}, t_{5}\right)=\left(x_{5}, x_{5} t_{5}\right)=(x, y), \quad \sigma_{5}\left(u_{5}, y_{5}\right)=\left(u_{5} y_{5}, y_{5}\right)=(x, y),
$$


and

$$
\begin{aligned}
& \sigma_{5}^{*}\left(\left(80 y-60 x y-80 x^{2}\right) d x-\left(y+32 x-36 x^{2}\right) d y\right) \\
& \quad=x_{5} \cdot\left[\left(-32 x_{5}-x_{5} t_{5}+36 x_{5}^{2}\right) d t_{5}+\left(48 t_{5}-80 x_{5}-24 x_{5} t_{5}-t_{5}^{2}\right) d x_{5}\right],
\end{aligned}
$$

that is, $l\left(\mathscr{H}_{2}, p_{5}\right)=1$.

The singularities of the transformed foliation along $E_{5}:=\sigma_{5}^{-1}\left(p_{5}\right)$ are a reduced singularity at $q_{5}:=(0,48)=\left(x_{5}, t_{5}\right)\left(\right.$ with Camacho-Sad index $-\frac{5}{3}$ relative to $\left.E_{5}\right)$ and a non-reduced singularity at $(0,0)=\left(x_{5}, t_{5}\right)$.

The blowing up $\sigma_{6}$ at $p_{6}:=(0,0)=\left(x_{5}, t_{5}\right)$ is written in local charts as

$$
\sigma_{6}\left(x_{6}, t_{6}\right)=\left(x_{6}, x_{6} t_{6}\right)=\left(x_{5}, y_{5}\right), \quad \sigma_{6}\left(u_{6}, y_{6}\right)=\left(u_{6} y_{6}, y_{2}\right)=\left(x_{5}, y_{5}\right),
$$

and

$$
\begin{aligned}
& \sigma_{6}^{*}\left[\left(-x_{5} t_{5}-32 x_{5}+36 x_{5}^{2}\right) d t_{5}+\left(48 t_{5}-24 x_{5} t_{5}-80 x_{5}-t_{5}^{2}\right) d x_{5}\right] \\
& \quad=x_{6} \cdot\left[\left(-80+16 t_{6}+12 t_{6} x_{6}-2 x_{6} t_{6}^{2}\right) d x_{6}+\left(-32 x_{6}+36 x_{6}^{2}-t_{6} x_{6}^{2}\right) d t_{6}\right],
\end{aligned}
$$

that is $l\left(\mathscr{H}_{2}, p_{6}\right)=1$.

There is a reduced singularity at $(0,0)=\left(u_{6}, y_{6}\right)$ which is the intersection $\overline{E_{5}} \cap E_{6}$ (with Camacho-Sad index $-\frac{1}{3}$ relative to $\overline{E_{5}}$ ). The non-reduced singularity of the transformed foliation along $E_{6}$ is at $(0,5)=\left(x_{6}, t_{6}\right)$.

After the linear change of coordinates $\left(x_{6}, t_{6}\right) \mapsto\left(x_{6}, t_{6}-5\right)$, the foliation around $p_{7}$ is induced by

$$
\left(16 t_{6}+10 x_{6}-8 x_{6} t_{6}-2 x_{6} t_{6}^{2}\right) d x_{6}+\left(-32 x_{6}+31 x_{6}^{2}-x_{6}^{2} t_{6}\right) d t_{6}=0 .
$$

The blowing up $\sigma_{7}$ at $p_{7}$ is written in local charts as

$$
\sigma_{7}\left(x_{7}, t_{7}\right)=\left(x_{7}, x_{7} t_{7}\right)=\left(x_{6}, y_{6}\right), \quad \sigma_{7}\left(u_{7}, y_{7}\right)=\left(u_{7} y_{7}, y_{7}\right)=\left(x_{6}, y_{6}\right),
$$

and

$$
\begin{gathered}
\sigma_{7}^{*}\left[\left(-2 x_{6} t_{6}^{2}-8 x_{6} t_{6}+10 x_{6}+16 t_{6}\right) d x_{6}+\left(-x_{6}^{2} t_{6}+31 x_{6}^{2}-32 x_{6}\right) d t_{6}\right] \\
=x_{7} \cdot\left[\left(10-16 t_{7}+26 x_{7} t_{7}-2 x_{7}^{2} t_{7}-x_{7}^{2} t_{7}^{2}\right) d x_{7}\right. \\
\left.+\left(-32 x_{7}+31 x_{7}^{2}-x_{7}^{3} t_{7}\right) d t_{7}\right],
\end{gathered}
$$

that is $l\left(\mathcal{H}_{2}, p_{7}\right)=1$.

There is a reduced singularity at $q_{7}:=\left(0, \frac{5}{8}\right)=\left(x_{7}, t_{7}\right)$ (with Camacho-Sad index -2 relative to $\left.E_{7}\right)$. The non-reduced singularity of the transformed foliation is at $(0,0)=\left(u_{7}, y_{7}\right) \in E_{7}$, where the foliation is induced by

$$
\left(-16 u_{7}+10 u_{7}^{2}-3 u_{7}^{2} y_{7}^{2}+23 u_{7}^{2} y_{7}^{2}\right) d y_{7}+\left(16 y_{7}+10 u_{7} y_{7}-8 u_{7} y_{7}^{2}-2 u_{7} y_{7}^{3}\right) d u_{7},
$$


which is clearly a dicritical singularity of radial type; that is, $l\left(\mathscr{H}_{2}, p_{8}\right)=2$.

Now let us reduce the singularity of $\mathscr{H}_{2}$ in the projective plane at $\left(\frac{32}{27}, \frac{1024}{81}\right)=$ $(x, y)$. The blowing up $\sigma_{9}$ at $p_{9}=\left(\frac{32}{27}, \frac{1024}{81}\right)$ produces two singularities along $E_{9}=\sigma_{9}^{-1}\left(p_{9}\right)$; a reduced singularity at $q_{9}:=\left(0, \frac{80}{3}\right)=\left(x_{9}, t_{9}\right)$ with index -3 relative to $E_{1}$ and a non-reduced singularity at $\left(0, \frac{320}{9}\right)=\left(x_{9}, t_{9}\right)$. It can be easily verified that $l\left(\mathscr{H}_{2}, p_{9}\right)=1$.

Next, the blow up $\sigma_{10}$ at $p_{10}:=\left(0, \frac{320}{9}\right)=\left(x_{9}, t_{9}\right)$ produces two singularities along $E_{10}=\sigma_{10}^{-1}\left(p_{10}\right)$. One is a reduced singularity at $q_{10}:=(0,105)=\left(x_{10}, t_{10}\right)$ with index -2 relative to $E_{10}$ and the other is a non-reduced singularity at infinity $(0,0)=\left(u_{10}, y_{10}\right)$. It is easily verified that also $l\left(\mathscr{H}_{2}, p_{10}\right)=1$. At last, it can be verified that $p_{11}:=(0,0)=\left(u_{10}, y_{10}\right)$ is a dicritical point of radial type, that is, $l\left(\mathscr{H}_{2}, p_{11}\right)=2$.

Finally, at the point $(1,4)=(x, y)$ in the projective plane, $\mathscr{H}_{2}$ has a reduced singularity. The quotient of eigenvalues of the linear part of a vector field inducing it is equal to $\frac{-3+\sqrt{5}}{2}$.

B.2. Resolution of $\mathscr{H}_{3}$. We begin with the singularity at infinity in the projective plane, which has the more involved resolution.

The foliation $\mathscr{H}_{3}$ is induced in affine coordinates $(x, y)$ of the plane by

$$
\Omega=\left(-\frac{5}{4} y^{2}+20 x y-60 x^{3}\right) d x+\left(-y+\frac{3}{4} x y+x^{2}\right) d y=0 .
$$

In the chart at infinity $(u, v)=\left(\frac{1}{x}, \frac{y}{x}\right)$ there is no singularity, as can be easily verified. The foliation $\mathscr{H}_{3}$ is induced at $p_{1}:=(0,0)=(w, s)=\left(\frac{x}{y}, \frac{1}{y}\right)$ by

$\Omega(w, s)=\left(-\frac{5}{4} s^{2}+20 w s^{2}-60 w^{3} s\right) d w+\left(\frac{1}{2} w s-21 w^{2} s+60 w^{4}+s^{2}\right) d s=0$,

where $s=0$ is an affine equation of the $\mathscr{H}_{3}$-invariant line at infinity.

The blowing up $\sigma_{1}$ at $p_{1}$ is written in local charts as

$$
\sigma_{1}\left(x_{1}, t_{1}\right)=\left(x_{1}, x_{1} t_{1}\right)=(w, s), \quad \sigma_{1}\left(u_{1}, y_{1}\right)=\left(u_{1} y_{1}, y_{1}\right)=(w, s),
$$

and

$\sigma_{1}^{*}(\Omega(w, s))=x_{1}^{2} \cdot\left[\left(-\frac{3}{4} t_{1}^{2}-x_{1} t_{1}^{2}+t_{1}^{3}\right) d x_{1}+\left(\frac{1}{2} x_{1} t_{1}-21 x_{1}^{2} t_{1}+60 x_{1}^{3}+x_{1} t_{1}^{2}\right) d t_{1}\right]$,

that is, $l\left(\mathscr{H}_{3}, p_{1}\right)=2$.

The singularities of the transformed foliation along $E_{1}:=\sigma_{1}^{-1}\left(p_{1}\right)$ are at $(0,0)=$ $\left(x_{1}, t_{1}\right)$ and $\left(0, \frac{3}{4}\right)=\left(x_{1}, t_{1}\right)$. The point $r_{1}:=\left(0, \frac{3}{4}\right)=\left(x_{1}, t_{1}\right)$ is a reduced singularity (with CS index equal to $-\frac{5}{3}$ relative to $E_{1}$ ). 
The blowing up $\sigma_{2}$ at $p_{2}:=(0,0)=\left(x_{1}, t_{1}\right)$ is written in local charts as

$$
\sigma_{2}\left(x_{2}, t_{2}\right)=\left(x_{2}, x_{2} t_{2}\right)=\left(x_{1}, y_{1}\right), \quad \sigma_{2}\left(u_{2}, y_{2}\right)=\left(u_{2} y_{2}, y_{2}\right)=\left(x_{1}, y_{1}\right),
$$

and

$$
\begin{gathered}
\sigma_{2}^{*}\left[\left(-\frac{3}{4} t_{1}^{2}-x_{1} t_{1}^{2}+t_{1}^{3}\right) d x_{1}+\left(\frac{1}{2} x_{1} t_{1}-21 x_{1}^{2} t_{1}+60 x_{1}^{3}+x_{1} t_{1}^{2}\right) d t_{1}\right] \\
=x_{2}^{2} \cdot\left[\left(60 x_{2} t_{2}-\frac{1}{4} t_{2}^{2}-22 x_{2} t_{2}^{2}+2 x_{2} t_{2}^{3}\right) d x_{2}\right. \\
\left.+\left(\frac{1}{2} x_{2} t_{2}-21 x_{2}^{2} t_{2}+60 x_{2}^{2}+x_{2} t_{2}^{2}\right) d t_{2}\right]
\end{gathered}
$$

that is $l\left(\mathscr{H}_{3}, p_{2}\right)=2$.

The non-reduced singularity of the transformed foliation along $E_{2}$ is at $(0,0)=$ $\left(x_{2}, t_{2}\right)$. Also there is a reduced singularity at infinity $(0,0)=\left(u_{2}, y_{2}\right)=E_{2} \cap \overline{E_{1}}$ (with CS index $-\frac{1}{3}$ relative to $\overline{E_{1}}$ ).

The blowing up $\sigma_{3}$ at $p_{3}:=(0,0)=\left(x_{2}, t_{2}\right)$ is written in local charts as

$$
\sigma_{3}\left(x_{3}, t_{3}\right)=\left(x_{3}, x_{3} t_{3}\right)=\left(x_{2}, y_{2}\right), \quad \sigma_{3}\left(u_{3}, y_{3}\right)=\left(u_{3} y_{3}, y_{3}\right)=\left(x_{2}, y_{2}\right),
$$

and

$$
\begin{gathered}
\sigma_{3}^{*}\left[\left(60 x_{2} t_{2}-\frac{1}{4} t_{2}^{2}-22 x_{2} t_{2}^{2}+2 x_{2} t_{2}^{3}\right) d x_{2}+\left(\frac{1}{2} x_{2} t_{2}-21 x_{2}^{2} t_{2}+60 x_{2}^{2}+x_{2} t_{2}^{2}\right) d t_{2}\right] \\
=x_{3}^{2} \cdot\left[\left(60 x_{3}+\frac{1}{2} x_{3} t_{3}-21 x_{3}^{2} t_{3}+x_{3}^{3} t_{3}^{2}\right) d t_{3}\right. \\
\left.\quad+\left(120 t_{3}+\frac{1}{4} t_{3}^{2}-43 x_{3} t_{3}^{2}+3 x_{3}^{2} t_{3}^{2}\right) d x_{3}\right]
\end{gathered}
$$

that is, $l\left(\mathscr{H}_{3}, p_{3}\right)=2$.

There is a reduced singularity at $r_{3}:=(0,-480)=\left(x_{3}, t_{3}\right) \in E_{3}$ and a reduced singularity at $(0,0)=\left(x_{3}, t_{3}\right)$ which is the crossing point with $\bar{L}$ (with Camacho-Sad index -2 relative to $\bar{L})$. The point at infinity in $E_{3}$, that is, $p_{4}:=(0,0)=\left(u_{3}, y_{3}\right)$ is a non- reduced singularity, where the foliation is induced by

$$
\left(\frac{-1}{4} y_{3}+60 u_{3} y_{3}-22 y_{3}^{2} u_{3}+2 u_{3} y_{3}^{2}\right) d u_{3}+\left(\frac{1}{4} u_{3}-43 u_{3}^{2} y_{3}+3 u_{3}^{2} y_{3}^{2}+120 u_{3}^{2}\right) d y_{3}=0,
$$

which clearly is a dicritical point of radial type, that is, $l\left(\mathscr{H}_{3}, p_{4}\right)=2$.

Let us now reduce the singularity of $\mathscr{H}_{3}$ in the projective plane at $p_{5}:=(0,0)=$ $(x, y)$. The blowing up $\sigma_{5}$ at $(0,0)$ is written in local charts as

$$
\sigma_{5}\left(x_{5}, t_{5}\right)=\left(x_{5}, x_{5} t_{5}\right)=(x, y), \quad \sigma_{5}\left(u_{5}, y_{5}\right)=\left(u_{5} y_{5}, y_{5}\right)=(x, y),
$$

and, since $\mathscr{H}_{3}$ is given by

$$
\Omega(x, y)=\left(-\frac{5}{4} y^{2}+20 x y-60 x^{3}\right) d x+\left(-y+\frac{3}{4} x y+x^{2}\right) d y=0,
$$


we have

$\sigma_{5}^{*}(\Omega(x, y))=x_{5} \cdot\left[\left(x_{5}^{2}+\frac{3}{4} x_{5}^{2} t_{5}-x_{5} t_{5}\right) d t_{5}+\left(21 x_{5} t_{5}-\frac{1}{2} x_{5} t_{5}^{2}-60 x_{5}^{2}-t_{5}^{2}\right) d x_{5}\right]$,

that is $l\left(\mathscr{H}_{3}, p_{5}\right)=1$.

The singularity of the transformed foliation along $E_{5}:=\sigma_{5}^{-1}\left(p_{5}\right)$ is just $(0,0)=$ $\left(x_{5}, t_{5}\right)$. The blowing up $\sigma_{6}$ at $p_{6}:=(0,0)=\left(x_{5}, t_{5}\right)$ is written in local charts as

$$
\sigma_{6}\left(x_{6}, t_{6}\right)=\left(x_{6}, x_{6} t_{6}\right)=\left(x_{5}, y_{5}\right), \quad \sigma_{6}\left(u_{6}, y_{6}\right)=\left(u_{6} y_{6}, y_{2}\right)=\left(x_{5}, y_{5}\right),
$$

and

$$
\begin{gathered}
\sigma_{6}^{*}\left[\left(x_{5}^{2}+\frac{3}{4} x_{5}^{2} t_{5}-x_{5} t_{5}\right) d t_{5}+\left(21 x_{5} t_{5}-\frac{1}{2} x_{5} t_{5}^{2}-60 x_{5}^{2}-t_{5}^{2}\right) d x_{5}\right] \\
=x_{6}^{2} \cdot\left[\left(-60+22 t_{6}+\frac{1}{4} x_{6} t_{6}^{2}-2 t_{6}^{2}\right) d x_{6}\right. \\
\left.\quad+\left(x_{6}+\frac{3}{4} x_{6}^{2} t_{6}-x_{6} t_{6}\right) d t_{6}\right]
\end{gathered}
$$

that is $l\left(\mathcal{H}_{2}, p_{6}\right)=2$. There is a reduced singularity at $r_{6}:=(0,6)=\left(u_{6}, y_{6}\right)$ (with Camacho-Sad index $-\frac{5}{2}$ relative to $E_{6}$ ). Also there is a reduced singularity at the intersection $(0,0)=\left(u_{6}, y_{6}\right)=\overline{E_{5}} \cap E_{6}$ (with Camacho-Sad index -2 relative to $\left.\overline{E_{5}}\right)$. Also there is a non-reduced singularity of the transformed foliation at $(0,5)=\left(x_{6}, t_{6}\right) \in E_{6}$.

After the linear change of coordinates $\left.\left(x_{6}, t_{6}\right) \mapsto\left(x_{6}, t_{6}-5\right)\right)$, the foliation is induced by

$$
\begin{aligned}
\eta:= & \left(2 t_{6}+\frac{25}{4} x_{6}+\frac{1}{4} x_{6} t_{6}^{2}-2 t_{6}^{2}+\frac{10}{4} x_{6} t_{6}\right) d x_{6} \\
& +\left(-4 x_{6}-x_{6} t_{6}+\frac{75}{4} x_{6}^{2}+\frac{3}{4} x_{6}^{2} t_{6}^{2}+\frac{30}{4} x_{6}^{2} t_{6}\right) d t_{6} .
\end{aligned}
$$

The blowing up $\sigma_{7}$ at $p_{7}:=(0,5)=\left(x_{6}, t_{6}\right)$ is written locally as

$$
\sigma_{7}\left(x_{7}, t_{7}\right)=\left(x_{7}, x_{7} t_{7}\right)=\left(x_{6}, y_{6}\right), \quad \sigma_{7}\left(u_{7}, y_{7}\right)=\left(u_{7} y_{7}, y_{7}\right)=\left(x_{6}, y_{6}\right),
$$

and

$$
\begin{gathered}
\sigma_{7}^{*} \eta=x_{7} \cdot\left[\left(\frac{15}{4}-2 t_{7}+\frac{75}{4} x_{7}+\frac{3}{2} x_{7} t_{7}+\frac{31}{4} x_{7}^{2} t_{7}^{2}-2 x_{7} t_{7}^{2}+\frac{3}{4} x_{7}^{3} t_{7}^{3}\right) d x_{7}\right. \\
\left.+\left(-4 x_{7}+\frac{75}{4} x_{7}^{2}-x_{7}^{2} t_{7}+\frac{30}{4} x_{7}^{3} t_{7}+\frac{3}{4} x_{7}^{4} t_{7}^{2}\right) d t_{7}\right]
\end{gathered}
$$

that is, $l\left(\mathscr{H}_{3}, p_{7}\right)=1$.

There is a reduced singularity at $\left(0, \frac{-25}{8}\right)=\left(x_{7}, t_{7}\right)$ (with Camacho-Sad index -2 relative to $E_{7}$ ). The non-reduced singularity of the transformed foliation is at 
$(0,0)=\left(u_{7}, y_{7}\right)$, where the foliation is induced by

$$
\begin{aligned}
\left(-2 u_{7}-3 u_{7} y_{7}+\frac{50}{4} u_{7}^{2}+\frac{85}{4} u_{7}^{2} y_{7}+\frac{3}{4} u_{7}^{2} y_{7}^{3}+\frac{31}{4} u_{7}^{2} y_{7}^{2}\right) d y_{7} \\
+\left(2 y_{7}-2 y_{7}^{2}+\frac{25}{4} u_{7} y_{7}+\frac{10}{4} u_{7} y_{7}^{2}+\frac{1}{4} u_{7} y_{7}^{3}\right) d u_{7},
\end{aligned}
$$

which is clearly a dicritical singularity of radial type; that is, $l\left(\mathscr{H}_{3}, p_{8}\right)=2$.

Let us reduce the singularity of $\mathscr{H}_{3}$ in the projective plane at $\left(\frac{32}{27}, \frac{1024}{81}\right)=(x, y)$. The blowing up $\sigma_{9}$ at $p_{9}=\left(\frac{32}{27}, \frac{1024}{81}\right)$ produces two singularities along $E_{9}=$ $\sigma_{9}^{-1}\left(p_{9}\right)$; a reduced singularity at $r_{9}:=(0,0)=\left(x_{9}, t_{9}\right)$ with index -3 relative to $E_{1}$ and a non-reduced singularity at $\left(0, \frac{320}{9}\right)=\left(x_{9}, t_{9}\right)$. It can be easily verified that $l\left(\mathscr{H}_{3}, p_{9}\right)=1$.

Next, the blow up $\sigma_{10}$ at $p_{10}:=\left(0, \frac{320}{9}\right)=\left(x_{9}, t_{9}\right)$ produces two singularities along $E_{10}=\sigma_{10}^{-1}\left(p_{10}\right)$. One is a reduced singularity at $(0,25)=\left(x_{10}, t_{10}\right)$ with index -2 relative to $E_{10}$, the other a non-reduced singularity at infinity $(0,0)=$ $\left(u_{10}, y_{10}\right)$. It is easily verified that $l\left(\mathscr{H}_{3}, p_{10}\right)=1$. At last, it is easily verified that $p_{11}:=(0,0)=\left(u_{10}, y_{10}\right)$ is a dicritical point of radial type, that is, $l\left(\mathscr{H}_{3}, p_{11}\right)=2$.

At the point $(1,4)=(x, y)$ in the projective plane, $\mathscr{H}_{3}$ has a reduced singularity. The quotient of eigenvalues of the linear part of a vector field inducing it is given by $\frac{-3+\sqrt{5}}{2}$.

\section{References}

[1] J.Artés, B. Grünbaum and J. Llibre, On the number of invariant straight lines for polynomial differential systems. Pacific J. Math. 184 (2) (1998), 207-230. Zbl 0930.34022 MR 1628583

[2] L. Badescu, Algebraic surfaces. Universitext, Springer-Verlag, New York 2001. Zbl 0965.14001 MR 1805816

[3] L. Bayle and A. Beauville, Birational involutions of $\mathbb{P}^{2}$. Kodaira's Issue. Asian J. Math. 4 (2000), 11-17. Zbl 01585205 MR 1802909

[4] M. Brunella, Birational geometry of foliations. Monografias de Matemática, Instituto de Matemática Pura e Aplicada (IMPA), Rio de Janeiro 2000. Zbl 01893057 MR 1948251

[5] M. Brunella, Subharmonic variation of the leafwise Poincaré metric. Invent. Math 152 (2003), 119-148. Zbl 1029.32014 MR 1965362

[6] H. S. M. Coxeter, Regular polytopes. Methuen \& Co., Ltd., London; Pitman Publishing Corporation, New York 1948. Zbl 0031.06502 MR 0027148

[7] G. van der Geer, Hilbert modular surfaces. Ergeb. Math. Grenzgeb. (3) 16, Springer-Verlag, Berlin 1988. Zbl 0634.14022 MR 0930101

[8] L. Godeaux, L'involution de Geiser. Bull. Soc. Math. Belgique 1952 (1952), 4-7. Zbl 0051.37802 MR 0058239 
Vol. 80 (2005)

[9] F. Hirzebruch, The Hilbert modular group for the field $Q(\sqrt{5})$, and the cubic diagonal surface of Clebsch and Klein. Russian Math. Surveys 31 (5) (1976), 96-110. Zbl 0356.14010 MR 0498397

[10] F. Hirzebruch, The ring of Hilbert modular forms for real quadratic fields in small discriminant. In Modular functions of one variable, VI, Lecture Notes in Math. 627, SpringerVerlag, Berlin 1977, 287-323. Zbl 0369.10017 MR 0480355

[11] R. Kobayashi, K. Kushibiki and I. Naruki, Polygons and Hilbert Modular Groups. Tôhoku Math. J. 41 (1989), 633-646. Zbl 0693.10020 MR 1025329

[12] M. McQuillan, Non-Commutative Mori Theory. Preprint (revised version), IHES, 2001 (available at www . ihes.fr).

[13] L. G. Mendes, Kodaira dimension of holomorphic singular foliations. Bol. Soc. Brasil. Mat. (N.S.) 31 (2000), 127-143. Zbl 0979.32017 MR 1785264

[14] T. A. Schmidt, Klein's cubic surface and a non-arithmetic curve. Math. Ann. 309 (1997), 533-539. Zbl 0885.11033 MR 1483822

[15] F. Sakai, $D$-dimension of algebraic surfaces and numerically effectiver divisors. Compositio Math. 48 (1983), 101-118. Zbl 0509.14038 MR 0700582

[16] B. Scárdua, Transversely affine and transversely projective holomorphic foliations. Ann. Sci. École Norm. Sup. (4) 30 (2) (1997), 169-204 Zbl 0889.32031 MR 1432053

[17] F. Touzet, Sur les feuilletages holomorphes transversalment projectives. Ann. Inst. Fourier 53 (3) (2003), 815-846. Zbl 1032.32020 MR 2008442

Received May 23, 2003

L. G. Mendes, J. V. Pereira, Instituto de Matemática Pura e Aplicada, Est. D. Castorina, 110, 22460-320, Rio de Janeiro, RJ, Brazil

E-mail: mendes@mat.ufrgs.br, jvp@impa.br 